\title{
Shape deformations in rough-surface scattering: improved algorithms
}

\author{
David P. Nicholls \\ Department of Mathematics, University of Notre Dame, Notre Dame, Indiana 46556 \\ Fernando Reitich \\ School of Mathematics, University of Minnesota, Minneapolis, Minnesota 55455
}

Received February 21, 2003; revised manuscript received August 27, 2003; accepted November 14, 2003

\begin{abstract}
We present new, stabilized shape-perturbation methods for calculations of scattering from rough surfaces. For practical purposes, we present new algorithms for both low- (first- and second-) and high-order implementations. The new schemes are designed with guidance from our previous results that uncovered the basic mechanism behind the instabilities that can arise in methods based on shape perturbations [D. P. Nicholls and F. Reitich, J. Opt. Soc. Am. A 21, 590 (2004)]. As was shown there, these instabilities stem from significant cancellations that are inevitably present in the recursions underlying these methods. This clear identification of the source of instabilities resulted also in a collection of guiding principles, which we now test and confirm. As predicted, improved low-order algorithms can be attained from an explicit consideration of the recurrence. At high orders, on the other hand, the complexity of the formulas precludes an explicit account of cancellations. In this case, however, the theory suggests a number of alternatives to implicitly mollify them. We show that two such alternatives, based on a change of independent variables and on Dirichlet-to-interior-derivative operators, respectively, successfully resolve the cancellations and thus allow for very-high-order calculations that can significantly expand the domain of applicability of shape-perturbation approaches. (C) 2004 Optical Society of America

OCIS codes: $050.0050,050.2770,290.0290$.
\end{abstract}

\section{INTRODUCTION}

Shape-perturbation methods for scattering calculations have a long history ${ }^{1,2}$ and their simplicity, efficiency, and accuracy have rendered them invaluable tools in a wide array of applications. Both low-order ${ }^{2-9}$ and highorder $^{10-20}$ expansions have been investigated and implemented numerically with varied degrees of success. This variation can be largely attributed to the limited understanding of some fundamental properties of these methods, specifically those related to convergence and conditioning, that existed until very recently. Indeed, the issue of convergence of shape-perturbation series generated a historical controversy dating to the 1950s and only recently resolved. ${ }^{21}$ Issues of stability, on the other hand, had remained largely unexplored until our recent work in Ref. 22, where we discussed the conditioning properties of boundary perturbation schemes. In particular, in that paper we presented a theory that explains the observed behavior of these methods, whose performance typically deteriorates with increasing surface roughness and/or increasing number of discretization points beyond a threshold. The theory attributes this behavior to significant cancellations in the recursions underlying these numerical procedures, and it further provides some guidelines for the design of new, stabilized implementations. Here we demonstrate that the principles derived from the theories in Ref. 22 can indeed be carried out to generate shape-deformation algorithms with improved conditioning properties.

As argued in Ref. 22, different strategies can be fol- lowed depending on whether low- or high-order perturbation expansions are considered. For the sake of broad applicability, we shall discuss both cases, since, as we said, both low- and high-order expansions have been, and continue to be, used in a variety of contexts. For low- (first-, second-) order implementations, we shall show that, as anticipated in Ref. 22, the relative simplicity of the recurrences does allow for an explicit account of cancellations. More precisely, we shall explain how the formulas can be recast in a manner that explicitly avoids the calculation of small numbers (e.g., high-frequency Fourier coefficients) as differences of large ones. For higher-order simulations, on the other hand, this strategy becomes increasingly complex, as the formulas get progressively more complicated. As indicated in Ref. 22, however, cancellations in these cases can be implicitly accounted for. Specifically, we shall show that improved recursions can be obtained if care is taken in avoiding differentiation of the fields across the boundaries of their domain of definition. To this end, we propose two different strategies, both motivated by the results in Ref. 22, to improve the performance of the field expansion (FE) and operator expansion (OE) methods, respectively. ${ }^{22}$ The first approach improves upon the FE method by means of a change of independent variables, effected a priori of the perturbation expansion, which guarantees differentiations inside the domain of definition of the fields. In the case of the $\mathrm{OE}$ approach, on the other hand, these inner differentiations are secured by replacing the standard perturbative calculation of Dirichlet-to-Neumann operators (DNOs) by one 
that computes the DNO of the surface of interest by embedding it in a family of Dirichlet-to-interior-derivative operators (DIDOs); see Subsection 3.B.

The rest of the paper is organized as follows. First, in Section 2 we address the conditioning of low-order implementations. For this, we begin from basic reformulations of the $\mathrm{OE}$ and $\mathrm{FE}$ recurrences as derived in Ref. 22, which we further develop here to attain stabilized implementations. Section 3 then is devoted to high-order expansions. There we present our proposals for improvements in the FE (Subsection 3.A) and OE (Subsection 3.B) implementations as described above. Finally, concluding remarks follow in Section 4.

\section{LOW-ORDER METHODS}

In this section, we present our approach to the design of low-order shape-deformation methods with improved stability properties. As we said, our basic strategy consists of an explicit recasting of the underlying recursive relations for the successive terms in the perturbation series in a way that prevents all consequential cancellations. This basic premise then is clearly applicable to all standard perturbative algorithms. Here we shall exemplify this in the context of the well-known operator expansion (OE) and field expansion (FE) schemes, which we briefly review next.

A. Classical Methods: Operator and Field Expansions As with all shape-deformation methods, the standard method of field expansions (also known by a variety of alternative names, including Small-Perturbation Method, Rayleigh-Fourier method, Rayleigh-Rice theory, iterative series solution, etc.) exploits the explicit solvability of the scattering problem in the case of a flat surface by proposing a perturbation series expansion for the field scattered by a general profile. More precisely, for a plane wave

$$
v_{\text {inc }}(x, y)=\exp (i \alpha x-i \beta y)
$$

incident on a $d$-periodic profile $y=g(x)$, the scattered field $v(x, y)$ is sought in the form

$$
v(x, y)=\sum_{n=0}^{\infty} v_{n}(x, y)
$$

where

$$
v_{n}=\mathcal{O}\left(g^{n}\right) .
$$

If the surface is perfectly conducting, for instance, a recurrence relation can be derived from the boundary condition

$$
v(x, g(x))=-\exp [i \alpha x-i \beta g(x)] .
$$

Indeed, with the use of Eq. (2.2), it follows that the functions $v_{n}$ satisfy the sequence of scattering problems ${ }^{22}$

$$
\begin{aligned}
& \Delta v_{n}+k^{2} v_{n}=0, \\
& v_{n}(x, 0)=P_{n}(x), \\
& \partial_{y} v_{n}(x, a)+T(a)\left[v_{n}(\cdot, a)\right](x)=0,
\end{aligned}
$$

$$
v_{n}(x+d, y)=\exp (i \alpha d) v_{n}(x, y)
$$

for $0<y<a$, where

$$
\begin{aligned}
P_{n}(x)= & -\exp (i \alpha x) \frac{[-i \beta g(x)]^{n}}{n !} \\
& -\sum_{l=0}^{n-1} \frac{[g(x)]^{n-l}}{(n-l) !} \partial_{y}^{n-l} v_{l}(x, 0),
\end{aligned}
$$

so that Eqs. (2.4) provide a recursive definition of $v_{n}$. Here Eq. (2.4d) reflects the periodicity of the structure, and Eq. (2.4c) expresses Sommerfeld's radiation condition for the scattered field in terms of a Dirichlet-to-Neumann operator (DNO) (see Ref. 22). More precisely, for any surface $y=\sigma(x), T(\sigma)$, is an operator that acts on (quasiperiodic) functions $\xi(x)$ defined on $y=\sigma(x)$ : The operator maps the function $\xi$ onto a function $T(\sigma)[\xi]$, also defined on the surface, according to

$$
T(\sigma)[\xi](x)=\frac{\partial \Xi}{\partial n(x)}(x, \sigma(x)),
$$

where $n(x)=\left(\partial_{x} \sigma(x),-1\right)$ is normal to the surface and $\Xi=\Xi(x, y)$ solves the scattering problem with boundary data $\xi(x)$ on $y=\sigma(x)$ [that is, $\Xi(x, \sigma(x))=\xi(x)$ ]. Note that, in particular, for a flat surface $y=a$ the DNO can be explicitly written as

$$
T(a)\left[\sum_{p=-\infty}^{\infty} \hat{\xi}_{p} \exp \left(i \alpha_{p} x\right)\right]=\sum_{p=-\infty}^{\infty}\left(-i \beta_{p}\right) \hat{\xi}_{p} \exp \left(i \alpha_{p} x\right),
$$

where

$$
\alpha_{p}=\alpha+\frac{2 \pi}{d} p, \quad \beta_{p}=\sqrt{k^{2}-\alpha_{p}^{2}}
$$

As has been shown by a number of authors, Eqs. (2.4) then provide an efficient means to approximate the scattered field, as they simply entail the recursive evaluation of a sequence of scattering problems off a flat surface, which can be effectively attained through fast Fourier transforms (FFTs) (see Ref. 22 and the references therein).

A similar situation arises in connection with the $\mathrm{OE}$ method, ${ }^{23-29}$ which constitutes an alternative method for the prediction of scattering returns based on shape deformations. The approach is based on two simple observations: First, we note that Green's identity for the field $v$ scattered off a surface $y=g(x)$ can be written as

$$
\begin{aligned}
v(x, y)= & \int_{0}^{d}\left\{-\frac{\partial \Phi}{\partial n\left(x^{\prime}\right)}\left(x-x^{\prime}, y-g\left(x^{\prime}\right)\right) f_{\text {inc }}\left(x^{\prime}\right)\right. \\
& \left.+\Phi\left(x-x^{\prime}, y-g\left(x^{\prime}\right)\right) T(g)\left[f_{\text {inc }}\right]\left(x^{\prime}\right)\right\} \mathrm{d} x^{\prime}
\end{aligned}
$$

where $\Phi(x, y)$ denotes the (outgoing, time-harmonic) free-space Green's function, $T(g)$ is the DNO on $y$ $=g(x)$, and $f_{\text {inc }}$ denotes the incident field evaluated on the surface. Obviously, all quantities in Eq. (2.8) are explicit save for that involving the DNO, which is typically 
difficult to find. The second central observation in the OE approach then relates to the DNO itself, and it is based on the explicit nature that the operator takes on for a flat surface. Indeed, the explicit formula (2.6) suggests an efficient method for the evaluation of the DNO corresponding to an arbitrary surface $y=g(x)$, namely through a perturbation series

$$
T(g)[\xi]=\sum_{n=0}^{\infty} T_{n}(g)[\xi]
$$

about a flat surface $y=0$, so that $T_{n}(g)=\mathcal{O}\left(g^{n}\right)$. In fact, a formal expansion readily delivers the recurrence ${ }^{22}$

$$
\begin{aligned}
T_{n}(g)[\xi]= & \left(i \beta_{D}\right)^{n-1}\left\{\partial_{x} \frac{[g(x)]^{n}}{n !} \partial_{x} \xi+k^{2} \frac{[g(x)]^{n}}{n !} \xi\right\} \\
& -\sum_{l=0}^{n-1}\left(i \beta_{D}\right)^{n-l} \frac{[g(x)]^{n-l}}{(n-l) !} T_{l}(g)[\xi], \quad
\end{aligned}
$$

whose implementation then constitutes the core of the $\mathrm{OE}$ procedure. Here the (pseudo-differential) operator $\beta_{D}$ is defined as

$$
\beta_{D}=i T(0)=-i \partial_{y}
$$

or, with the use of (2.6),

$$
\beta_{D}\left[\sum_{p} \hat{\xi}_{p} \exp \left(i \alpha_{p} x\right)\right]=\sum_{p} \beta_{p} \hat{\xi}_{p} \exp \left(i \alpha_{p} x\right) .
$$

Therefore Eq. (2.10) can be solved very efficiently, as it entails only multiplication and (pseudo-) differentiation of (quasi-) periodic functions, which can again be accelerated with the use of FFTs.

We close this subsection by noting that when one is interested in the scatter off a specific profile $y=g(x)$ [and not on that generated by intermediate surfaces $y$ $=\delta_{g}(x)$, a particularly appealing feature of the OE procedure is that only the DNO is approximated perturbatively; in particular, the function $f_{\text {inc }}$ in Eq. (2.8) is not expanded in powers of $g(x)$. This, in turn, suggests an alternative to the FE scheme ${ }^{22}$ in Eqs. (2.4) wherein the incoming wave is not expanded in powers of $g(x)$. More precisely, the convergence of the series (2.1) can be accelerated if Eq. (2.4b) is replaced by

$$
\begin{aligned}
v_{n}(x, 0)= & -\delta_{n, 0} \exp \left[i \alpha x^{\prime}-i \beta g\left(x^{\prime}\right)\right] \\
& -\sum_{l=0}^{n-1} \frac{[g(x)]^{n-l}}{(n-l) !} \partial_{y}^{n-l} v_{l}(x, 0) .
\end{aligned}
$$

With this addition, we shall refer to the new scheme as a partial field expansion (PFE) and use it to (more fairly) compare with the OE method.

\section{B. Cancellations}

As argued in Ref. 22, the origins of the instabilities in the $\mathrm{OE}$ and FE schemes are rather apparent from Eqs. (2.10), and (2.12). Indeed, for instance, Eqs. (2.7) imply that

$$
\beta_{p} \approx i \frac{2 \pi}{d}|p|, \quad|p| \gg 1,
$$

so that the operator $\beta_{D}$ acts essentially as differentiation. It follows then that the individual terms on the righthand side of Eq. (2.10) contain derivatives of the profile $g(x)$ (and of the data $\xi$ ) of increasingly high order. On the other hand, classical results in the theory of singular integrals $^{30,31}$ guarantee that the series in Eq. (2.9) converges for rough (Lipschitz) profiles that may not even possess a single derivative in a classical sense. As explained in Ref. 22, this apparent contradiction can be reconciled only if, as it happens, all high-order derivatives appearing on the right-hand side of Eq. (2.10) exactly cancel out. Similar cancellations occur in Eq. (2.12), though, in this case, high-order differentiation is inherently present in the recursion and must be properly accounted for. $^{22}$ In any case, within a numerical implementation, these cancellations are, of course, a recipe for illconditioning, as it amounts to calculating relatively small numbers (e.g., the Fourier coefficients of $\left.T_{n}(g)[\xi]\right)$ as a difference of very large numbers [e.g., the Fourier coefficients of each (singular) individual term on the righthand side of Eq. (2.10)].

As we have shown, ${ }^{22}$ at low (first, second, third) order of the recurrences (2.10) and (2.12), the cancellations can be explicitly identified. Indeed, for $n=1,2$, formula (2.10) can be rewritten as

$$
\begin{aligned}
& T_{1}(g)[\xi]=-(D g)(D \xi)-R_{g}\left[\beta_{D} \xi\right], \\
& T_{2}(g)[\xi]=i \beta_{D} S_{g}\left[\beta_{D} \xi\right],
\end{aligned}
$$

while, if $n=2,3$, Eq. (2.12) is equivalent to

$$
\begin{aligned}
& v_{2}=-\beta_{D}\left[\frac{g^{2}}{2} \beta_{D} \xi\right]-S_{g}\left[\beta_{D} \xi\right], \\
& v_{3}=i \beta_{D}^{2}\left[\frac{g^{3}}{6} \beta_{D} \xi\right]+i g \beta_{D} S_{g}\left[\beta_{D} \xi\right] .
\end{aligned}
$$

The operators $R_{f}$ and $S_{f}$ are (nonconvolution) singular integral operators defined by

$$
\begin{aligned}
& R_{f}[\eta]=\beta_{D}[f \eta]-f \beta_{D}[\eta], \\
& S_{f}[\eta]=-R_{f^{2} / 2}[\eta]+f R_{f}[\eta],
\end{aligned}
$$

respectively. These operators can be shown to possess certain "smoothing" properties (see Appendix A), which, in turn, imply that Eqs. (2.13) and (2.14) account for all significant cancellations in the original recurrences.

\section{Improved Low-Order Algorithms}

As discussed above, the observations in Ref. 22 allow for the identification of the main source of instabilities in typical shape-perturbation schemes. Here we shall use this insight in the design of new and improved implementations, which we first address in the context of low-order calculations. Our effort in this direction is motivated in part by the popularity of such implementations, which, in turn, is partly due to their simplicity. For our purposes, this simplicity translates into the possibility of explicitly accounting for cancellations, as described in Subsection 2.B. Indeed, in view of these results, our objective reduces to providing stable numerical implementations for the relations (2.13) and (2.14). We shall pursue this here 
through the orders indicated there, although the general philosophy of Ref. 22 is not restricted, in principle, to the second- and third-order calculations that we present below. At higher orders, however, analogous calculations, though possible, become quite involved. For high-order calculations, on the other hand, alternative procedures, which will be presented in Section 3, obviate the need for an explicit management of cancellations.

As we said, our goal here is the design of stable implementations of Eqs. (2.13) and (2.14). To this end, we begin by noting that the regularizing character of the operators $R_{f}$ and $S_{f}$ in Eqs. (2.15) and (2.16) is apparent only in physical space, where they take on the form ${ }^{22}$

$$
\begin{aligned}
& R_{f}[\eta](x)=\frac{1}{d} \int_{0}^{d} \eta(y)[f(y)-f(x)] Q(x-y) \mathrm{d} y, \\
& S_{f}[\eta](x)=-\frac{1}{2 d} \int_{0}^{d} \eta(y) Q(x-y)[f(y)-f(x)]^{2} \mathrm{~d} y,
\end{aligned}
$$

where

$$
\begin{aligned}
Q(x) & \equiv \sum_{p=-\infty}^{\infty} \beta_{p} \exp \left(i \alpha_{p} x\right) \\
& \approx-\frac{i \exp (i \alpha x)}{2 \sin ^{2}(\pi x / d)} \text { as } x \rightarrow 0
\end{aligned}
$$

is a singular kernel. These representations, however, are perhaps not optimal for numerical calculations, as they entail the design of quadratures for (hyper)singular integrals. An alternative approach is to resort to the frequency domain representations of these operators, which read as

$$
\begin{aligned}
\widehat{R_{f}[\eta]_{p}}= & \sum_{q}\left(\beta_{p}-\beta_{p-q}\right) \hat{f}_{q} \hat{\eta}_{p-q}, \\
\widehat{S_{f[}[\eta]_{p}}= & \sum_{r} \sum_{q} \hat{f}_{r} \hat{f}_{q} \hat{\eta}_{p-r-q}\left[\left(\beta_{p-q}-\beta_{p-r-q}\right)\right. \\
& \left.-\left(\frac{1}{2} \beta_{p}-\frac{1}{2} \beta_{p-r-q}\right)\right] \\
= & \frac{1}{2} \sum_{r} \sum_{q} \hat{f}_{r} \hat{f}_{q} \hat{\eta}_{p-r-q}\left[\left(\beta_{p-q}-\beta_{p}\right)\right. \\
& \left.+\left(\beta_{p-q}-\beta_{p-r-q}\right)\right]
\end{aligned}
$$

on account of the definitions (2.15) and (2.16). Here, however, a straightforward implementation would bring back a significant proportion of the very cancellations that we are trying to avoid, as is clear from the multipliers $\beta_{p}-\beta_{p-q}$ and $\beta_{p-q}-\beta_{p-r-q}$ that appear in Eqs. (2.17) and (2.18) and that cancel out for large values of the indices. In the present, low-order context, however, these cancellations can again be explicitly rectified. Indeed, as we show in Appendix A, the relations (2.17) and (2.18) can be rewritten in the form

${\widehat{R_{f}[\eta]}}_{p}=\sum_{q} \frac{[-(2 \pi / d) q]\left(\alpha_{p}+\alpha_{p-q}\right)}{\beta_{p}+\beta_{p-q}} \hat{f}_{q} \hat{\eta}_{p-q}$,

$$
\begin{aligned}
{\widehat{S_{f}[\eta]}}_{p}= & \sum_{r} \sum_{q}\left[\frac{(2 \pi / d)^{2} q r}{\beta_{p}+\beta_{p-r-q}}\right] \\
& \times\left[1+\frac{\left(\alpha_{p}+\alpha_{p-q}\right)\left(\alpha_{p-q}+\alpha_{p-r-q}\right)}{\left(\beta_{p}+\beta_{p-q}\right)\left(\beta_{p-q}+\beta_{p-r-q}\right)}\right] \\
& \times \hat{f}_{r} \hat{f}_{q} \hat{\eta}_{p-r-q},
\end{aligned}
$$

where now the multipliers are void of cancellations.

Combining Eqs. (2.13) with Eqs. (2.19) and (2.20), we arrive at the (stable) OE formulas

$$
\begin{aligned}
\left(\widehat{T_{1}(g)[\xi]}\right)_{p}= & -\sum_{q}[(2 \pi / d) q] \hat{g}_{q} \alpha_{p-q} \hat{\xi}_{p-q} \\
& +\sum_{q} \frac{[(2 \pi / d) q]\left(\alpha_{p}+\alpha_{p-q}\right)}{\beta_{p}+\beta_{p-q}} \hat{g}_{q} \beta_{p-q} \hat{\xi}_{p-q}, \\
\left(\widehat{T_{2}(g)[\xi]}\right)_{q}= & i \beta_{p} \sum_{r} \sum_{q}\left[\frac{(2 \pi / d)^{2} q r}{\beta_{p}+\beta_{p-r-q}}\right] \\
& \times\left[1+\frac{\left(\alpha_{p}+\alpha_{p-q}\right)\left(\alpha_{p-q}+\alpha_{p-r-q}\right)}{\left(\beta_{p}+\beta_{p-q}\right)\left(\beta_{p-q}+\beta_{p-r-q}\right)}\right] \\
& \times \hat{g}_{r} \hat{g}_{q} \beta_{p-r-q} \hat{\xi}_{p-r-q} .
\end{aligned}
$$

Similarly, from Eqs. (2.14), we obtain new FE relations in the form

$$
\begin{aligned}
\left(\widehat{v_{2}}\right)_{p}= & -\frac{1}{2} \beta_{p} \sum_{r} \sum_{q} \hat{g}_{r} \hat{g}_{q} \beta_{p-r-q} \hat{\xi}_{p-r-q} \\
& -\sum_{r} \sum_{q}\left[\frac{(2 \pi / d)^{2} q r}{\beta_{p}+\beta_{p-r-q}}\right] \\
& \times\left[1+\frac{\left(\alpha_{p}+\alpha_{p-q}\right)\left(\alpha_{p-q}+\alpha_{p-r-q}\right)}{\left(\beta_{p}+\beta_{p-q}\right)\left(\beta_{p-q}+\beta_{p-r-q}\right)}\right] \\
& \times \hat{g}_{r} \hat{g}_{q} \beta_{p-r-q} \hat{\xi}_{p-r-q}, \\
\left(\widehat{v}_{3}\right)_{p}= & \frac{i}{6} \beta_{p}^{2} \sum_{r, q, s} \hat{g}_{r} \hat{g}_{q} \hat{g}_{s} \beta_{p-r-q-s} \hat{\xi}_{p-r-q-s} \\
& +i \sum_{r, q, s}\left[\frac{(2 \pi / d)^{2} s q}{\beta_{p-r}+\beta_{p-r-q-s}}\right] \beta_{p-r} \\
& \times\left[1+\frac{\left(\alpha_{p-r}+\alpha_{p-r-s}\right)\left(\alpha_{p-r-s}+\alpha_{p-r-q-s}\right)}{\left(\beta_{p-r}+\beta_{p-r-s}\right)\left(\beta_{p-r-s}+\beta_{p-r-q-s}\right)}\right] \\
& \times \hat{g}_{r} \hat{g}_{q} \hat{g}_{s} \beta_{p-r-q-s} \hat{\xi}_{p-r-q-s} .
\end{aligned}
$$

In Figs. 1 and 2, we present numerical results that illustrate the beneficial effect of using the stable formulas (2.22) in place of Eq. (2.10), and (2.24) in place of Eqs. (2.4) and (2.12) to compute the coefficients $\left(\widehat{T_{2}(g)[\xi]}\right)_{p}$ and $\left(\widehat{v_{3}}\right)_{p}$. The figures correspond to

$$
\hat{\xi}_{p}=p^{-2}, \quad \hat{g}_{p}=p^{-2}, \quad|p| \leqslant F,
$$

and a number $N_{x}=2048$ of discretization points (that is, wave numbers $p$ with $-1024 \leqslant p \leqslant 1023$ represented). To avoid aliasing effects and isolate the instabilities, we 


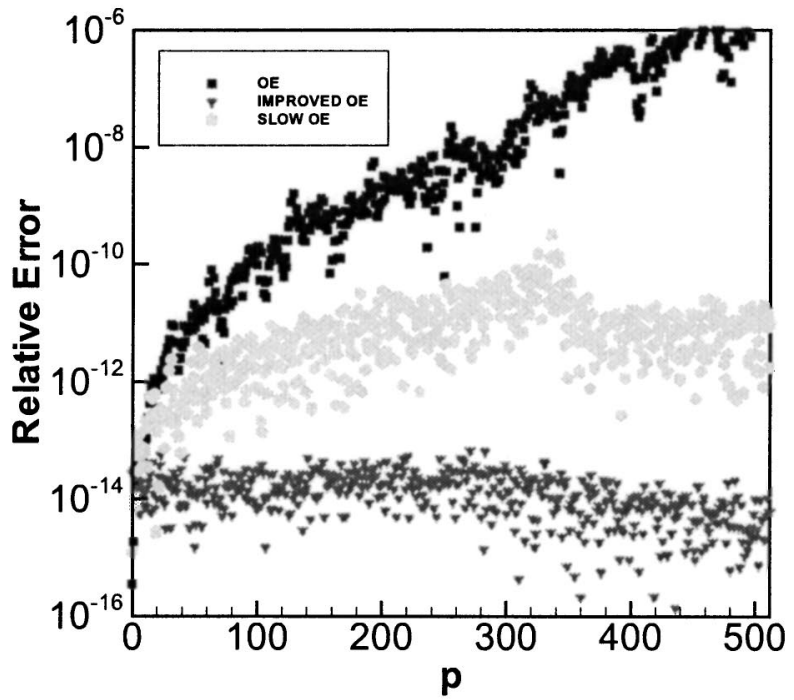

(a) $\alpha=\beta=1, k=\sqrt{2}$

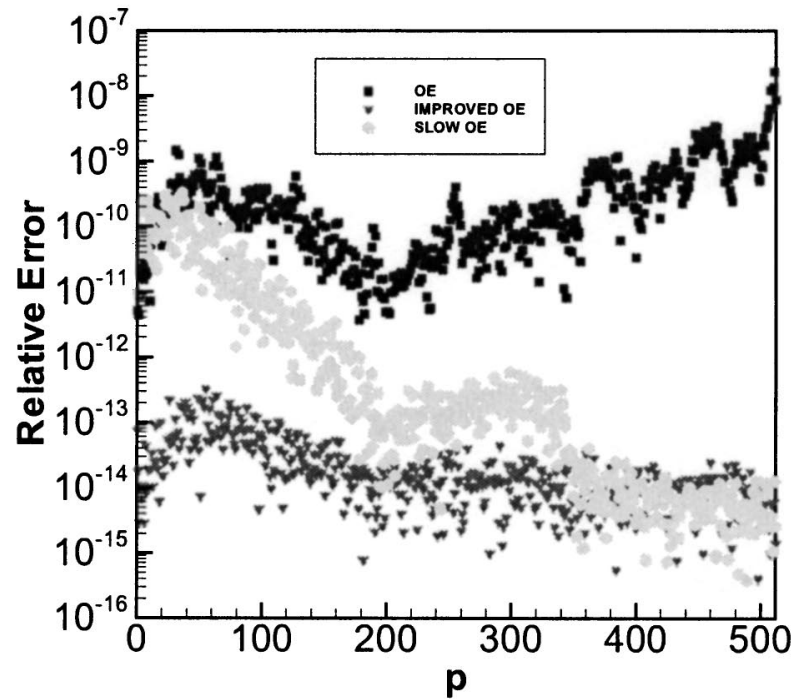

(b) $\alpha=0, \beta=200, k=200$

Fig. 1. Plots of relative error in absolute value of the Fourier coefficient $\left(\widehat{T_{2}(g)[\xi]}\right)_{p}$ for two different incident waves, computed by using $\mathrm{OE}$, Improved $\mathrm{OE}$, and Slow OE implementations.

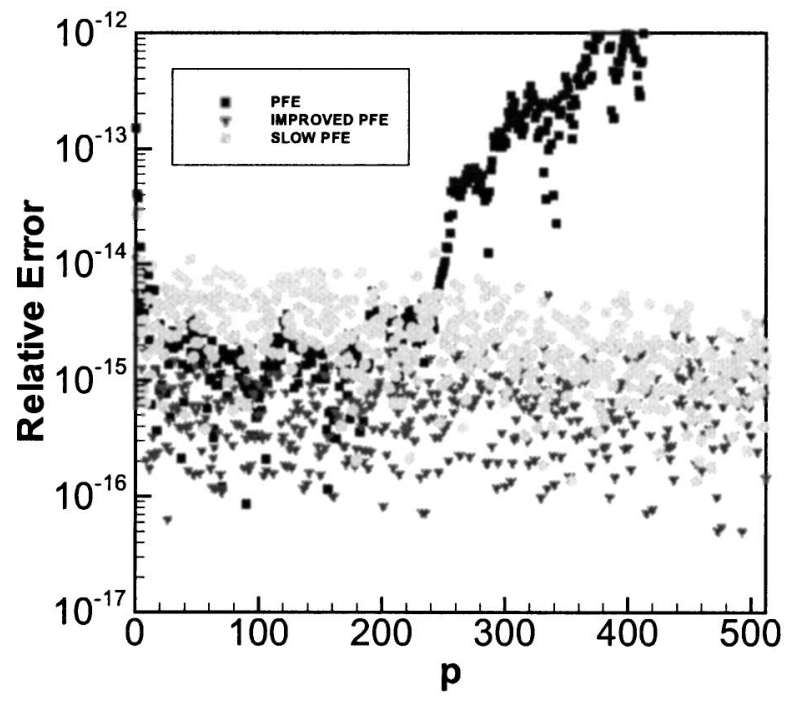

(a) $\alpha=\beta=1, k=\sqrt{2}$

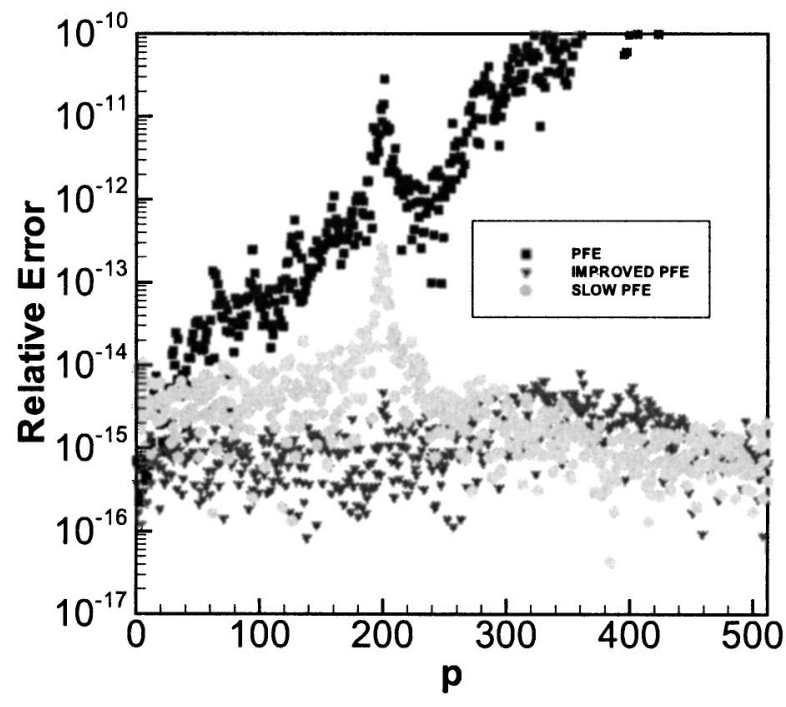

(b) $\alpha=0, \beta=200, k=200$

Fig. 2. Plots of relative error in absolute value of the Fourier coefficient $\left(\widehat{v_{3}}\right)_{p}$ for two different incident waves, computed by using PFE, Improved PFE, and Slow PFE implementations.

set $F=341$ and $F=256$ for the approximation of $T_{2}(g)[\xi]$ and $v_{3}(x, y)$, respectively. In addition to the new formulas (2.22) and (2.24) (labeled as Improved OE and Improved PFE, respectively), the figures also display the results obtained from two implementations of Eq. (2.10) and Eqs. (2.4) and (2.12): the standard one, using FFTs (labeled OE and PFE, respectively), and one where the convolutions in Fourier space are performed without FFT acceleration (labeled Slow OE and Slow PFE, respectively).

Our motivation to include the latter experiments is twofold. On the one hand, the results in Ref. 22 guarantee that slow convolutions will lead to better-conditioned numerics. Indeed, as explained there, the basic schemes lead to consideration of possibly very irregular functions (high-order derivatives of the surface and incidence), which, for efficiency, are multiplied in physical space and differentiated in the frequency domain. Clearly, as the regularity of these functions decreases (with increasing order of differentiation), this process becomes significantly ill-conditioned, as the passage through physical space relies on cancellations to approximate the functional values. In contrast, slow convolutions allow for the full calculation to remain in the frequency domain and therefore for the stable calculation of Fourier series. [An instructive example in this connection is provided by 
the product of two "delta functions" with pole at the origin, approximated in the frequency domain by a finite Fourier series with unit coefficients: A fast calculation would rely on the actual sum of the Fourier series, which, away from the origin, should produce exactly 0 as a sum of exponentials; in contrast, a slow convolution will deliver the (large) Fourier coefficients of the product directly and stably as a sum of 1s.] A second reason for including the slow calculations relates to the fact that a stable accelerated implementation of the formulas (2.22) and (2.24) is not immediate, so that a comparison with these, equally costly, Slow OE and Slow PFE procedures appears natural.

From the figures, we see that the new schemes significantly improve upon the quality of the approximations. Indeed, for instance, the predicted enhanced stability of the slow procedures is evident in the PFE case (Fig. 2). Interestingly, in the OE example this improvement is substantially less pronounced. In fact, this difference in behavior can again be explained on the basis of the findings in Ref. 22 , where a subtle distinction between these algorithms was first noted. Indeed, as explained there, all high-order derivatives of the profile and the incidence cancel out of formula (2.10), while these are inherently present in the recurrences (2.4) and (2.12). In view of this, it follows that Eq. (2.10) entails the calculation of $s u$ perfluous singularities that remain to be cancelled even if the calculation is entirely performed (slowly) in the frequency domain. In contrast, the addition of slow convolutions in an implementation of Eqs. (2.4) and (2.12) has a beneficial effect on the approximation of its (truly present) singular part. As a result, the gains from the further stabilized formulas (2.22) and (2.24) are more pronounced in relation to the $\mathrm{OE}$ approach (Fig. 1), where they bypass the fictitious cancellations of Eq. (2.10). Still, as shown in Fig. 2, for the PFE method the new formulas do provide further gains in accuracy, which should be attributed to the additional subtle rearrangement of the singular part of Eq. (2.12) as explained in Ref. 22, Subsection 3.B.

\section{HIGH-ORDER METHODS}

As we stated, an explicit rearrangement of the recursive formulas, as pursued in Section 2, could be attempted at higher orders. As is clear from the calculations in Appen$\operatorname{dix} \mathrm{A}$, however, this procedure becomes increasingly complex with increasing order. For this reason, we introduce in this section two alternative approaches, specifically designed for high-order computations, that attain their enhanced stability in an implicit manner. The first of these, described in Subsection 3.A, is motivated by the discussion of Ref. 22, where it was shown that a simple change of variables

$$
x^{\prime}=x, \quad y^{\prime}=a\left[\frac{y-g(x)}{a-g(x)}\right],
$$

mapping the shape of the scatterer $y=g(x)$ to the plane $y^{\prime}=0$, allows for a direct recursive estimation of the terms in the perturbation series of the field

$$
u\left(x^{\prime}, y^{\prime}\right)=\sum_{n=0}^{\infty} u_{n}\left(x^{\prime}, y^{\prime}\right), \quad u_{n}=\mathcal{O}\left(g^{n}\right),
$$

in the transformed variables. As argued in Ref. 22, the success of this iterative bounding procedure implies that, in contrast with the formulas in the original variables, the new recurrence for the terms $u_{n}$ in Eq. (3.2) does not entail significant cancellations. And this, in turn, suggests that its numerical implementation should lead to a stable algorithm. In Subsection 3.A, we show that this is indeed the case and that, in fact, this approach significantly improves the accuracy and the applicability of classical shape-deformation methods.

As we explain below, however, these gains are attained at the expense of an increase in computational cost. If we insist on computational times that are exactly comparable with those associated with standard algorithms (e.g., OE), a different approach may be necessary [although we expect that further research may produce a more efficient implementation of the recurrence for $u_{n}$ in Eq. (3.2) than the one we present below]. In this direction, we introduce in Subsection 3.B a novel algorithm that exhibits enhanced stability properties while retaining the computational complexity of the classical schemes. This algorithm is also motivated by our observations above, but it uses the theory indirectly to motivate an improvement over the $\mathrm{OE}$ procedure.

\section{A. Transformed Field Expansions}

As we have shown, ${ }^{22}$ the cancellations present in Eq. (2.12) preclude a straightforward estimation of the size of the functions $v_{n}$ in Eqs. (2.4) in a way that would guarantee the convergence of the series (2.1). On the other hand, we also showed there that this can be rectified by using the transformation (3.1). Indeed, letting

$$
u\left(x^{\prime}, y^{\prime}\right)=v\left(x^{\prime}, g\left(x^{\prime}\right)+\frac{a-g\left(x^{\prime}\right)}{a} y^{\prime}\right)
$$

and expressing this transformed field in the form of a perturbation series as in Eq. (3.2), one can derive a new set of recursive formulas, which read $\mathrm{as}^{22}$

$$
\begin{aligned}
& \Delta u_{n}+k^{2} u_{n}=\left(1-\delta_{n, 0}\right) F_{n}\left(x^{\prime}, y^{\prime}\right), \\
& u_{n}\left(x^{\prime}, 0\right)=-(-i \beta)^{n} \exp \left(i \alpha x^{\prime}\right) \frac{\left[g\left(x^{\prime}\right)\right]^{n}}{n !}, \\
& \partial_{y^{\prime}} u_{n}\left(x^{\prime}, a\right)+T(a)\left[u_{n}(\cdot, a)\right]\left(x^{\prime}\right)=R_{n}\left(x^{\prime}\right), \\
& u_{n}\left(x^{\prime}+d, y^{\prime}\right)=\exp (i \alpha d) u_{n}\left(x^{\prime}, y^{\prime}\right)
\end{aligned}
$$

for $0<y^{\prime}<a$, where $\delta_{n, j}$ is the Kronecker delta,

$$
\begin{aligned}
F_{n}\left(x^{\prime}, y^{\prime}\right)= & \operatorname{div}_{x^{\prime}}\left[F_{n}^{(1)}\left(x^{\prime}, y^{\prime}\right)\right]+\partial_{y^{\prime}} F_{n}^{(2)}\left(x^{\prime}, y^{\prime}\right) \\
& +F_{n}^{(3)}\left(x^{\prime}, y^{\prime}\right)
\end{aligned}
$$




$$
\begin{aligned}
& F_{n}^{(1)}\left(x^{\prime}, y^{\prime}\right)=\frac{2 g\left(x^{\prime}\right)}{a} \nabla_{x^{\prime}} u_{n-1}-\frac{\left[g\left(x^{\prime}\right)\right]^{2}}{a^{2}} \nabla_{x^{\prime}} u_{n-2} \\
& +\frac{\left(a-y^{\prime}\right) \nabla_{x^{\prime}} g\left(x^{\prime}\right)}{a} \partial_{y^{\prime}} u_{n-1} \\
& -\frac{\left(a-y^{\prime}\right) g\left(x^{\prime}\right) \nabla_{x^{\prime}} g\left(x^{\prime}\right)}{a^{2}} \partial_{y^{\prime}} u_{n-2}, \\
& F_{n}^{(2)}\left(x^{\prime}, y^{\prime}\right)=\frac{\left(a-y^{\prime}\right) \nabla_{x^{\prime}} g\left(x^{\prime}\right)}{a} \cdot \nabla_{x^{\prime}} u_{n-1} \\
& -\frac{\left(a-y^{\prime}\right) g\left(x^{\prime}\right) \nabla_{x^{\prime}} g\left(x^{\prime}\right)}{a^{2}} \cdot \nabla_{x^{\prime}} u_{n-2} \\
& -\frac{\left(a-y^{\prime}\right)^{2}\left|\nabla_{x^{\prime}} g\left(x^{\prime}\right)\right|^{2}}{a^{2}} \partial_{y^{\prime}} u_{n-2}, \\
& F_{n}^{(3)}\left(x^{\prime}, y^{\prime}\right)=-\frac{\nabla_{x^{\prime}} g\left(x^{\prime}\right)}{a} \cdot \nabla_{x^{\prime}} u_{n-1}+\frac{g\left(x^{\prime}\right) \nabla_{x^{\prime}} g\left(x^{\prime}\right)}{a^{2}} \\
& \nabla_{x^{\prime}} u_{n-2}+\frac{\left(a-y^{\prime}\right)\left|\nabla_{x^{\prime}} g\left(x^{\prime}\right)\right|^{2}}{a^{2}} \partial_{y^{\prime}} u_{n-2} \\
& +\frac{2 g\left(x^{\prime}\right) k^{2}}{a} u_{n-1}-\frac{\left[g\left(x^{\prime}\right)\right]^{2} k^{2}}{a^{2}} u_{n-2}, \\
& R_{n}\left(x^{\prime}\right)=\frac{g\left(x^{\prime}\right)}{a} T(a)\left[u_{n-1}(\cdot, a)\right]\left(x^{\prime}\right) .
\end{aligned}
$$

As demonstrated in Ref. 22, this new recurrence does not entail significant cancellations, as the successive iterates $u_{n}$ can be recursively estimated from Eqs. (3.4) by bounding the functions $F_{n}$ inductively with a direct use of the triangle inequality. This lack of cancellations, in turn, suggests that a numerical implementation of Eqs. (3.4), which we shall refer to as constituting the method of transformed field expansion (TFE), should be significantly better conditioned than that of Eqs. (2.4) and (2.12).

As we mentioned in Subsection 2.A in connection with the FE method, the convergence of the series (2.1) will be accelerated if Eq. (2.4b) is replaced by Eq. (2.12), i.e., the incoming wave is not expanded in powers of $g(x)$. The same comment applies to Eq. (3.2) in regard to the TFE algorithm, where Eq. (3.4b) can be replaced by

$$
u_{n}\left(x^{\prime}, 0\right)=-\delta_{n, 0} \exp \left[i \alpha x^{\prime}-i \beta g\left(x^{\prime}\right)\right] .
$$

We term this new method the partial transformed field expansion (PTFE) algorithm, which we will again use to compare with the $\mathrm{OE}$ and $\mathrm{PFE}$ algorithms. In fact, in the remainder of this section we present a collection of numerical examples that clearly illustrate the convergence properties of the OE and PFE schemes, as well as the significant improvements that can be attained with PTFE.

For the numerical implementation of the OE and PFE recursions, we shall use a spectral collocation method ${ }^{32}$ wherein

$$
\begin{aligned}
T_{n}(g)\left[f_{\mathrm{inc}}\right](x) & =\sum_{|p|<N_{x} / 2} \tau_{n, p} \exp \left(i \alpha_{p} x\right), \\
v_{n}(x, y) & =\sum_{|p|<N_{x} / 2} d_{n, p} \exp \left(i \alpha_{p} x+i \beta_{p} y\right)
\end{aligned}
$$

for coefficients $\tau_{n, p}$ and $d_{n, p}$ that are iteratively obtained by means of FFTs and fast convolutions. In the case of PTFE, on the other hand, the problem at $n$th order entails the solution of a scattering problem, for a flat surface, with a source $F_{n}\left(x^{\prime}, y^{\prime}\right)$, which, of course, prevents the use of (a basis of) exact solutions as in Eqs. (3.6) and (3.7) to reduce the dimensionality of the problem. Indeed, in this case, the additional transverse dimension $y^{\prime}$ must also be resolved numerically, with a consequent increase in computational cost. More precisely, for the PTFE implementation we shall appeal to a spectral Fourier/Chebyshev-tau method which posits an approximate solution of the form

$$
\widetilde{u}_{n}\left(x^{\prime}, y^{\prime}\right)=\sum_{|p|<N_{x} / 2} \sum_{l=0}^{N_{y}} \hat{u}_{n}(p, l) \exp \left(i p x^{\prime}\right) \mathcal{T}_{l}\left(\frac{2 y^{\prime}-a}{a}\right),
$$

where $\mathcal{T}_{l}(z)$ is the $l$ th Chebyshev polynomial. The resulting set of equations can be efficiently solved with the use of fast Fourier and Chebyshev transforms in conjunction with the fast elliptic solve outlined in Ref. 33, Section 10. Still, as we said, the computational effort associated with such a strategy will typically be greater than that resulting from implementations of OE and PFE based on Eqs. (3.6) and (3.7); as we show below, however, this increased cost may be compensated by a substantial increase in accuracy that can, in fact, allow for computations beyond the reach of the $\mathrm{OE}$ and $\mathrm{PFE}$ algorithms.

To test the performance of the methods, we shall consider both a smooth sinusoidal surface

$$
y=\frac{h}{2} \cos \left(\frac{2 \pi x}{d}\right)
$$

and an instance of a rather generic rough surface given by

$$
\begin{aligned}
y= & \frac{h}{2}\left[\cos \left(\frac{2 \pi x}{d}\right)+\frac{1}{8} \sin \left(\frac{6 \pi x}{d}\right)+\frac{1}{9} \cos \left(\frac{6 \pi x}{d}\right)\right. \\
& \left.+\frac{1}{16} \cos \left(\frac{8 \pi x}{d}\right)\right]
\end{aligned}
$$

see Fig. 3. For each of these, we compute the diffracted efficiencies $e_{p}$, defined as ${ }^{34}$

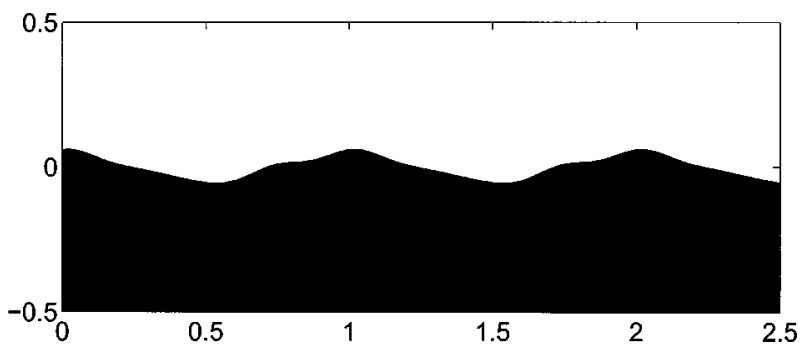

Fig. 3. Plot of the rough surface (3.10) $(d=1, h=0.1)$. 


$$
e_{p}=\frac{\beta_{p}}{\beta}\left|B_{p}\right|^{2},
$$

where $B_{p}$ denotes the $p$ th Rayleigh amplitude (see Ref. 22). For a lossless surface, the principle of conservation of energy yields

$$
\sum_{p \in U} e_{p}=1
$$

where

$$
U=\left\{p \mid \beta_{p}>0\right\}
$$

is the (finite) set of propagating modes. Equation (3.11) can be used to define a common measure of convergence of a numerical approximation, namely the energy error

$$
\varepsilon=\left|1-\sum_{p \in U} e_{p}\right|,
$$

which we shall use below. We note, however, that this test may not provide, in many instances, an accurate appraisal of the convergence properties of a numerical scheme for a number of reasons. On the one hand, clearly, a specific error in the sum in Eq. (3.12) does not guarantee a comparable error in each efficiency $e_{p}$. In addition, this test relates only to low-order efficiencies corresponding to propagating modes: High-order Rayleigh amplitudes (i.e., those for $p \notin U$ ) may be grossly miscalculated and still result in a small energy defect according to Eq. (3.12). In this connection, a more accurate assessment may be garnered, for instance, by comparison of exact and calculated normal derivatives of scattered fields (i.e., currents or tractions). In fact, a convenient approach to this end results if, for a given profile $y$ $=g(x)$, we consider an incident wave $v_{\text {inc }}$ satisfying

$$
v_{\text {inc }}(x, g(x))=\exp \left[i \alpha_{p} x+i \beta_{p} g(x)\right]
$$

for a fixed integer $p$, where $\alpha_{p}$ and $\beta_{p}$ are defined as in Eqs. (2.7). Indeed, in this case, we clearly have that the scattered field is given by

$$
v(x, y)=\exp \left(i \alpha_{p} x+i \beta_{p} y\right)
$$

and therefore the normal derivative on the surface is $e x$ plicitly expressed as

$$
\begin{aligned}
\frac{\partial v}{\partial n} & =\left.\left\{\left[\partial_{x} g(x)\right] \partial_{x}-\partial_{y}\right\} v\right|_{y=g(x)} \\
& =T(g)\left[\exp \left\{i \alpha_{p} x+i \beta_{p} g(x)\right\}\right] \\
& =\left[i \alpha_{p} \partial_{x} g(x)-i \beta_{p}\right] \exp \left[i \alpha_{p} x+i \beta_{p} g(x)\right],
\end{aligned}
$$

allowing for direct, near-field comparisons. Specifically, the implementation of the PFE, OE, and PTFE schemes to order $n$ provides approximate values $T_{n, N_{x}}^{\text {approx }}$ of this latter quantity at $N_{x}$ equally spaced nodes $x_{j}$ on $[0, d]$ for which we may define the error as

$$
\begin{aligned}
\text { Error } & =\operatorname{Error}\left(n, N_{x}\right) \\
& =\max _{1 \leqslant j \leqslant N_{x}}\left|T(g)[\xi]\left(x_{j}\right)-T_{n, N_{x}}^{\operatorname{approx}}\left(x_{j}\right)\right| ;
\end{aligned}
$$

Table 1. Energy Defect for the Sinusoidal Profile (3.9) under Normal Incidence with a

Wavelength-to-Period Ratio $\lambda=0.4368, d=1$, and a [32/32] Padé Approximant

\begin{tabular}{lccc}
\hline$h$ & \multicolumn{1}{c}{$\mathrm{PFE}$} & $\mathrm{PTFE}$ & $\mathrm{OE}$ \\
\hline 0 & 0 & 0 & 0 \\
0.05 & $3 \times 10^{-16}$ & $1 \times 10^{-16}$ & $3 \times 10^{-18}$ \\
0.1 & $1 \times 10^{-16}$ & $1 \times 10^{-16}$ & $8 \times 10^{-12}$ \\
0.15 & $6 \times 10^{-16}$ & $2 \times 10^{-15}$ & $2 \times 10^{-5}$ \\
0.2 & $1 \times 10^{-13}$ & $2 \times 10^{-15}$ & $5 \times 10^{-3}$ \\
0.25 & $4 \times 10^{-13}$ & $9 \times 10^{-15}$ & $3 \times 10^{-2}$ \\
0.3 & $3 \times 10^{-9}$ & $2 \times 10^{-15}$ & $6 \times 10^{-2}$ \\
0.35 & $4 \times 10^{-8}$ & $6 \times 10^{-14}$ & $1 \times 10^{-1}$ \\
0.4 & $1 \times 10^{-6}$ & $8 \times 10^{-12}$ & $1 \times 10^{-1}$ \\
0.45 & $3 \times 10^{-5}$ & $2 \times 10^{-10}$ & $2 \times 10^{-1}$ \\
0.5 & $2 \times 10^{-4}$ & $2 \times 10^{-8}$ & $2 \times 10^{-1}$ \\
0.55 & $3 \times 10^{-4}$ & $2 \times 10^{-7}$ & $4 \times 10^{-2}$ \\
0.6 & $7 \times 10^{-3}$ & $7 \times 10^{-6}$ & $4 \times 10^{-1}$ \\
0.65 & $2 \times 10^{-2}$ & $2 \times 10^{-6}$ & 2 \\
0.7 & $7 \times 10^{-2}$ & $2 \times 10^{-4}$ & $4 \times 10^{-1}$ \\
\hline
\end{tabular}

Table 2. Energy Defect for the Rough Surface (3.10) under Normal Incidence with a

Wavelength-to-Period Ratio $\lambda=0.4368, d=1$, and a [32/32] Padé Approximant

\begin{tabular}{lccc}
\hline \multicolumn{1}{c}{$h$} & $\mathrm{PFE}$ & $\mathrm{PTFE}$ & $\mathrm{OE}$ \\
\hline 0 & 0 & 0 & 0 \\
0.05 & $1 \times 10^{-15}$ & $1 \times 10^{-15}$ & $4 \times 10^{-4}$ \\
0.1 & $5 \times 10^{-12}$ & $3 \times 10^{-15}$ & $8 \times 10^{-3}$ \\
0.15 & $3 \times 10^{-9}$ & $2 \times 10^{-15}$ & $5 \times 10^{-5}$ \\
0.2 & $9 \times 10^{-8}$ & $5 \times 10^{-14}$ & $2 \times 10^{-2}$ \\
0.25 & $2 \times 10^{-5}$ & $6 \times 10^{-13}$ & $2 \times 10^{-2}$ \\
0.3 & $2 \times 10^{-4}$ & $3 \times 10^{-12}$ & $6 \times 10^{-2}$ \\
0.35 & $9 \times 10^{-4}$ & $7 \times 10^{-12}$ & $6 \times 10^{-2}$ \\
0.4 & $5 \times 10^{-4}$ & $1 \times 10^{-8}$ & $8 \times 10^{-2}$ \\
0.45 & $2 \times 10^{-2}$ & $1 \times 10^{-7}$ & $3 \times 10^{-4}$ \\
0.5 & $1 \times 10^{-1}$ & $4 \times 10^{-6}$ & $9 \times 10^{-2}$ \\
0.55 & 1 & $3 \times 10^{-5}$ & $1 \times 10^{-1}$ \\
0.6 & $4 \times 10^{-1}$ & $2 \times 10^{-4}$ & $1 \times 10^{-1}$ \\
\hline
\end{tabular}

see Fig. 11 below.

Still, in keeping with traditional measurements, we begin by presenting results in Eq. (3.12) for different heightto-period and wavelength-to-period ratios. The first set of results, in Tables 1 and 2, displays the energy defect for $\mathrm{OE}, \mathrm{PFE}$, and PTFE for a variety of heights $h$, wavelength $\lambda=0.4368 \quad$ (normal incidence), period $d$ $=1$, and profiles (3.9) and (3.10), respectively. In this case, there are five propagating modes $(U=\{p|| p$ $\mid \leqslant 2\}$ ). The parameters for the simulation in Table 1 are $N_{x}=64, N_{y}=48$, and $n=64$; for Table 2, they are $N_{x}=128, N_{y}=48$, and $n=64$. In both cases, the radiation condition (2.4c) and (3.4c) is imposed on $a=2$, and the perturbation series $(n=64)$ is summed by means of Padé approximation. ${ }^{35-37}$

As follows from the tables, PTFE provides a very significant improvement over $\mathrm{OE}$ and $\mathrm{PFE}$, allowing for accurate calculations beyond their domain of applicability. 
In fact, while the limit on the observed accuracy of PFE and $\mathrm{OE}$ is solely due to ill-conditioning, that of PTFE is due only to the limited resolution of the transverse direction $\left(N_{y}=48\right)$. These results are representative of the

Table 3. Energy Defect for the Rough Surface (3.10) under Normal Incidence with a

Wavelength-to-Period Ratio $\lambda=0.065, d=1$, and a [32/32] Padé Approximant

\begin{tabular}{lccc}
\hline \multicolumn{1}{c}{$h$} & PFE & PTFE & OE \\
\hline 0 & 0 & 0 & 0 \\
0.025 & $9 \times 10^{-16}$ & $1 \times 10^{-15}$ & $2 \times 10^{-16}$ \\
0.05 & $6 \times 10^{-14}$ & $3 \times 10^{-16}$ & $1 \times 10^{-3}$ \\
0.075 & $2 \times 10^{-11}$ & $2 \times 10^{-13}$ & $3 \times 10^{-3}$ \\
0.1 & $6 \times 10^{-8}$ & $1 \times 10^{-12}$ & $1 \times 10^{-2}$ \\
0.125 & $1 \times 10^{-5}$ & $6 \times 10^{-12}$ & $3 \times 10^{-3}$ \\
0.15 & $4 \times 10^{-4}$ & $1 \times 10^{-12}$ & $1 \times 10^{-3}$ \\
0.175 & $6 \times 10^{-3}$ & $3 \times 10^{-8}$ & $5 \times 10^{-4}$ \\
0.2 & $4 \times 10^{-1}$ & $7 \times 10^{-6}$ & $2 \times 10^{-4}$ \\
0.225 & 3 & $1 \times 10^{-4}$ & $2 \times 10^{-2}$ \\
0.25 & 6 & $2 \times 10^{-2}$ & $9 \times 10^{-2}$ \\
\hline
\end{tabular}

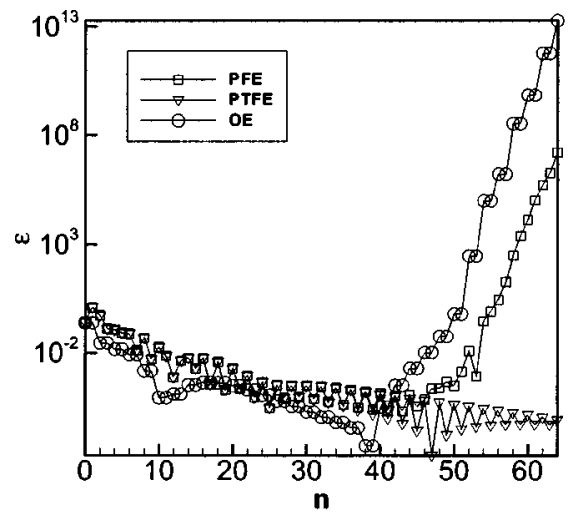

(a) Taylor overall behavior of the methods; additional results for the profile in Eq. (3.10) at a higher frequency of radiation (with $a$ now reduced to 0.2 ) corresponding to $\lambda=0.065$ ( $U=\{p|| p \mid \leqslant 15\})$ are presented in Table 3 .

Next, we present in Figs. 4-9 experiments that display the behavior of the methods as the order $n$ is increased. Several conclusions can be drawn from these figures (see also Refs. 38 and 39). First, the results show that PFE generally outperforms OE, while PTFE significantly improves on both. Moreover, as first noted in Refs. 36 and 37, the figures also show that Padé summation has a dramatic effect in accelerating the convergence of the Taylor series when it converges (e.g., Figs. 4, 6, and 8) and also in providing a convergent approximation when the Taylor series diverges (e.g., Figs. 5, 7, and 9). We also note that although the figures suggest that, for instance, the OE results continue to improve up to $n=20$ or even $n=30$, this holds only in the measure of energy defect (3.12), involving only low-frequency modes; the actual convergence of the individual, low-order modes is further verified in Fig. 10, where we check the convergence of specific efficiencies. On the other hand, we also show in Fig. 11 that the apparent improvement of the $\mathrm{OE}$ and PFE procedures

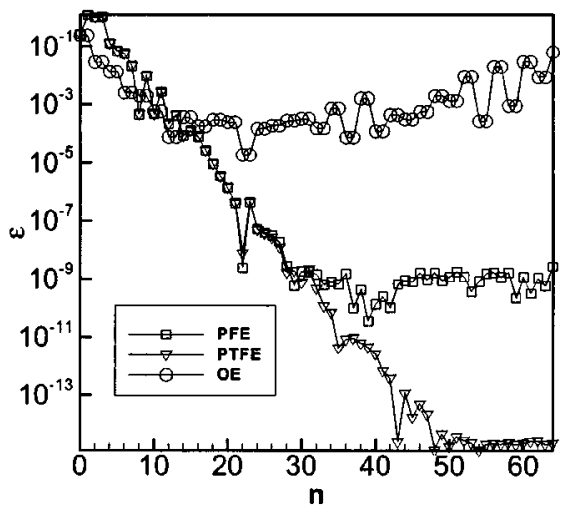

(b) Padé

Fig. 4. Energy defect (3.12) for a scattering configuration with the sinusoidal profile (3.9) using PFE, PTFE, and OE ( $\lambda / d$ $\left.=0.4368, h / d=0.3, d=1, a=2, N_{x}=64, N_{y}=48, n=64\right)$.

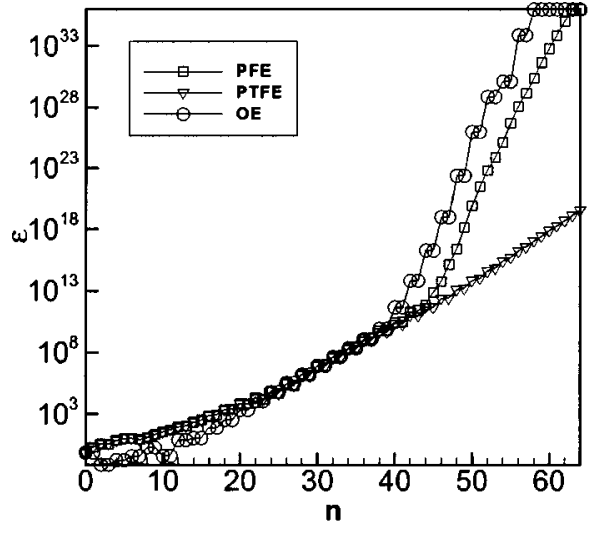

(a) Taylor

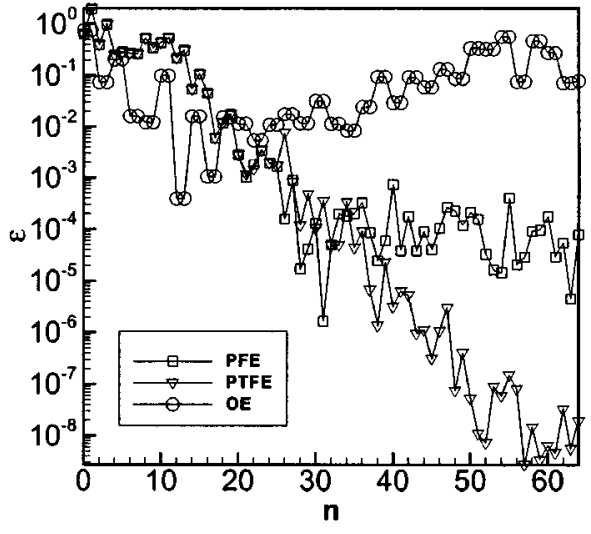

(b) Padé

Fig. 5. Energy defect (3.12) for a scattering configuration with the sinusoidal profile (3.9) using PFE, PTFE, and OE ( $\lambda / d$ $\left.=0.4368, h / d=0.5, d=1, a=2, N_{x}=64, N_{y}=48, n=64\right)$. 


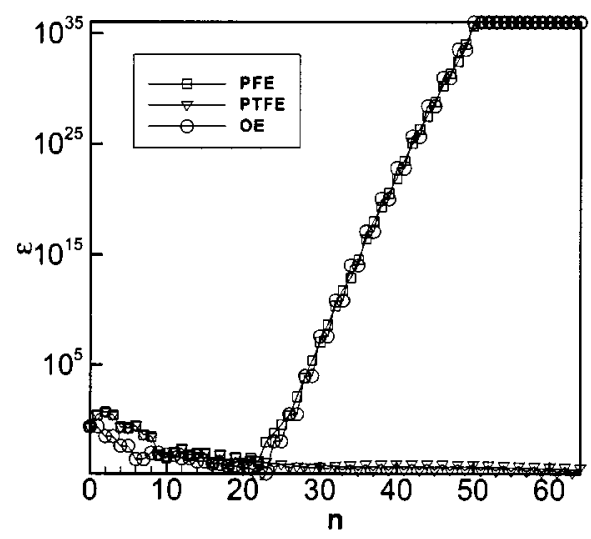

(a) Taylor

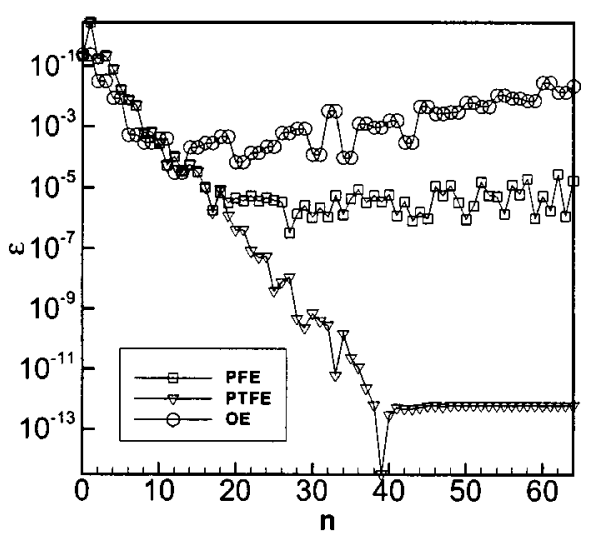

(b) Padé

Fig. 6. Energy defect (3.12) for a scattering configuration with the rough profile (3.10) using PFE, PTFE, and OE $(\lambda / d=0.4368, h / d$ $\left.=0.25, d=1, a=2, N_{x}=128, N_{y}=48, n=64\right)$.

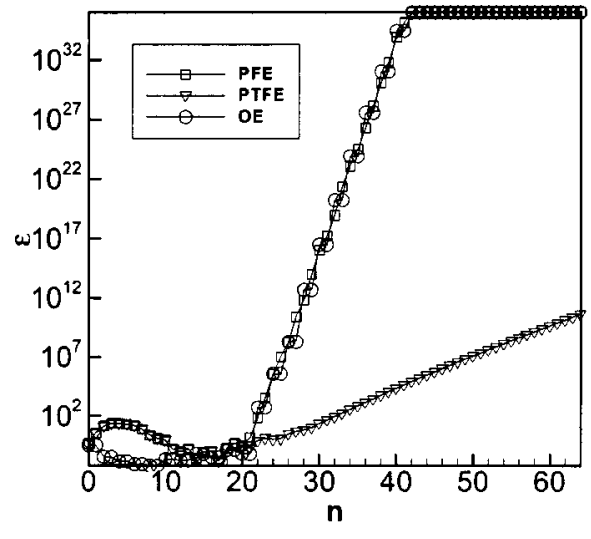

(a) Taylor

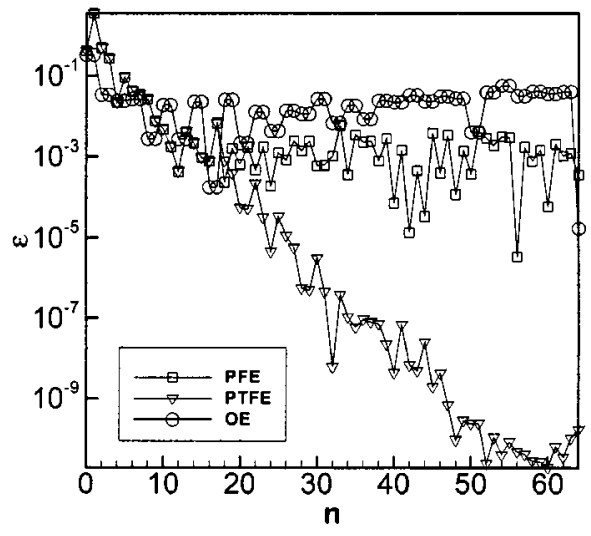

(b) Padé

Fig. 7. Energy defect (3.12) for a scattering configuration with the rough profile (3.10) using PFE, PTFE, and OE $(\lambda / d=0.4368, h / d$ $\left.=0.35, d=1, a=2, N_{x}=128, N_{y}=48, n=64\right)$.

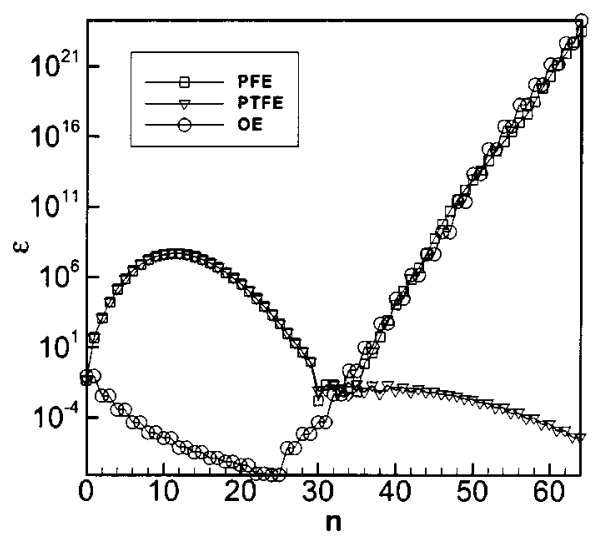

(a) Taylor

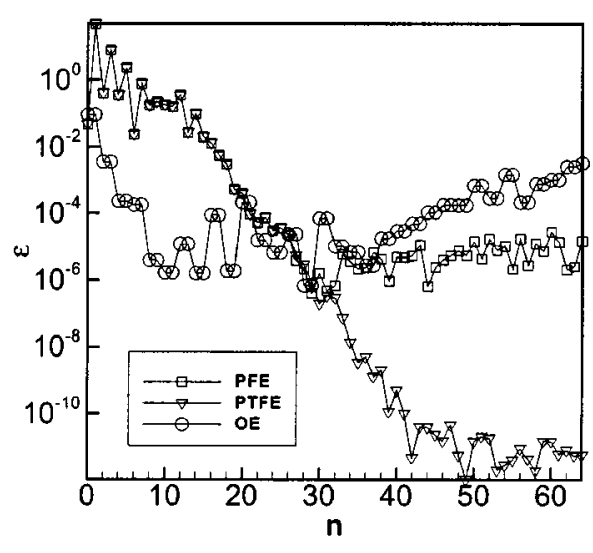

(b) Padé

Fig. 8. Energy defect (3.12) for a scattering configuration with the rough profile (3.10) using PFE, PTFE, and OE $(\lambda / d=0.065, h / d$ $\left.=0.125, d=1, a=0.2, N_{x}=128, N_{y}=48, n=64\right)$.

up to high orders is not realized in the near field. Indeed, as these results demonstrate (see also Figs. 12 and 13), and because of numerical instabilities arising from cancellations, no more than ten terms $(n \leqslant 10)$ in the OE recurrence can be accurately computed for a full approxima- tion of the currents. In any case, Figs. 10 and 11 show that the general conclusions on the stability of the different procedures suggested by the initial energy calculations do, in fact, still hold true (and, perhaps, even more so) for these more detailed analyses. 


\section{B. Dirichlet-to-Interior-Derivative Operators}

In light of the high-order calculations presented in Subsection 3.A, it is natural to ponder what lies at the root of the difference in the behavior of PTFE as contrasted with that of $\mathrm{OE}$ or PFE. In this connection, we contend that a

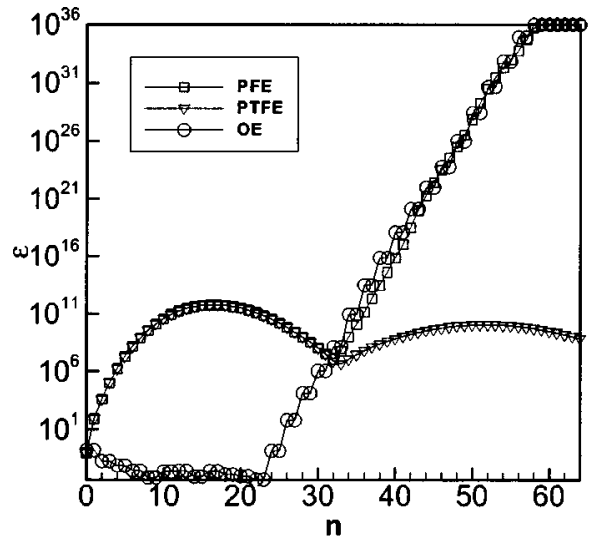

(a) Taylor main difference resides in the differentiations at the boundary that underlie all methods (in the derivation of the corresponding recursions). Indeed, we note that in the case of the $\mathrm{OE}$ and PFE schemes, these differentiations are performed across the boundary of definition of

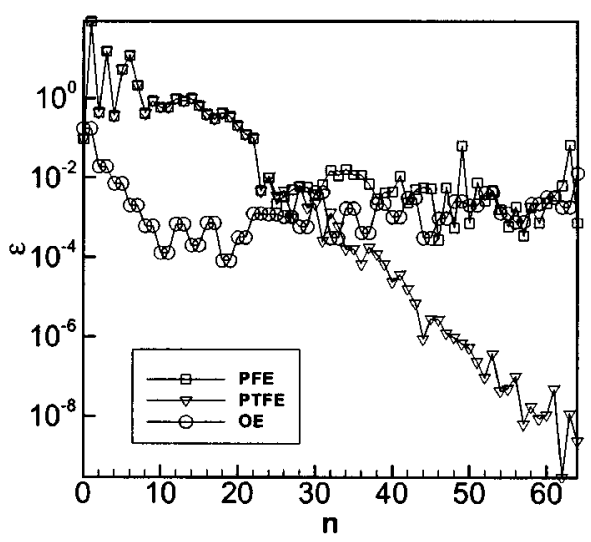

(b) Padé

Fig. 9. Energy defect (3.12) for a scattering configuration with the rough profile (3.10) using PFE, PTFE, and OE $(\lambda / d=0.065, h / d$ $\left.=0.175, d=1, a=0.2, N_{x}=128, N_{y}=48, n=64\right)$.

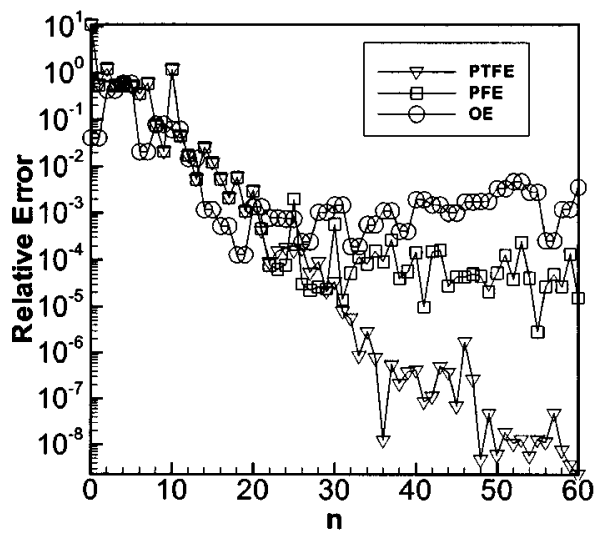

(a) Efficiency $e_{-15}$

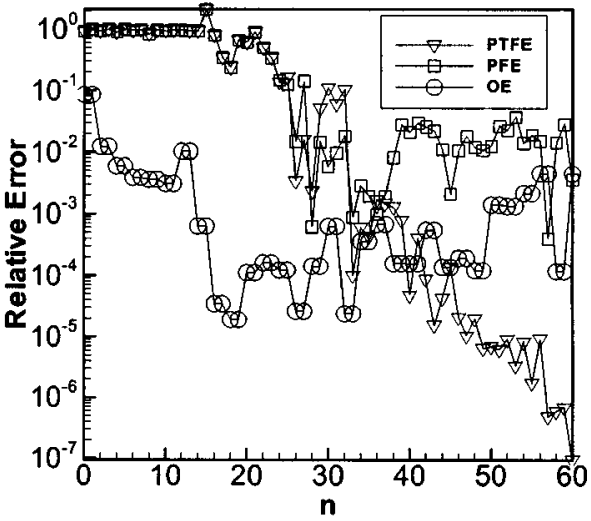

(b) Efficiency $e_{4}$

Fig. 10. Convergence of specific efficiencies $e_{-15}$ and $e_{4}$ for the configuration of Fig. 9 using PFE, PTFE, and OE; comparison of diagonal Padé approximations $([n / 2, n / 2])$ with an overresolved PTFE calculation ([32,32] approximant).

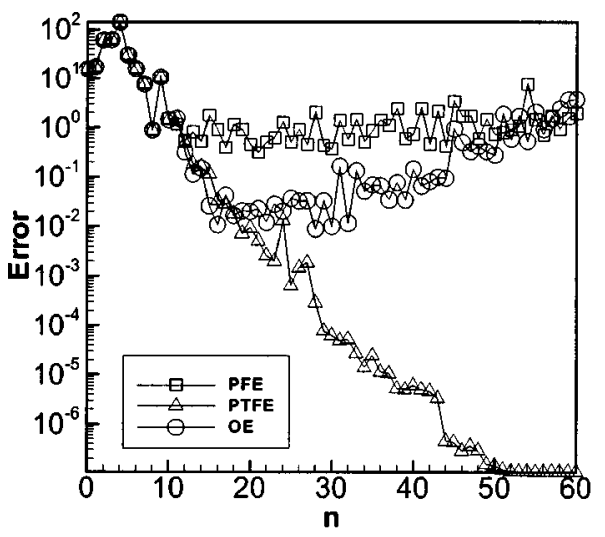

(a) $\lambda / d=0.4368, h / d=0.35$

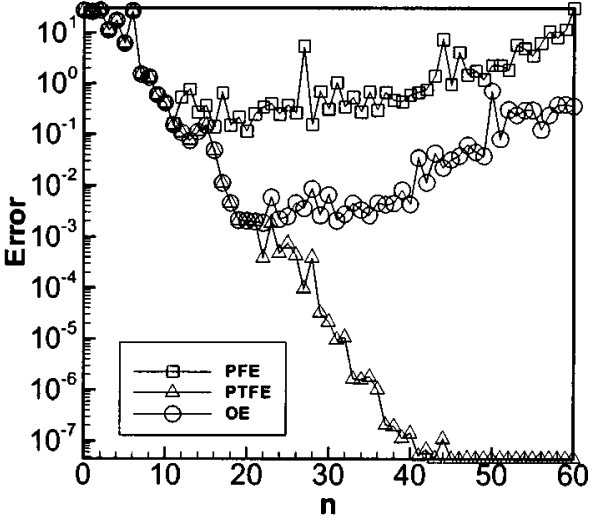

(b) $\lambda / d=0.065, h / d=0.175$

Fig. 11. Error (3.16) in the approximation of the current (using a diagonal Padé sum) for the configurations of (a) Fig. 7 and (b) Fig. 9 with incidence (3.13) and $p=1$; comparison with exact solution (3.15). 


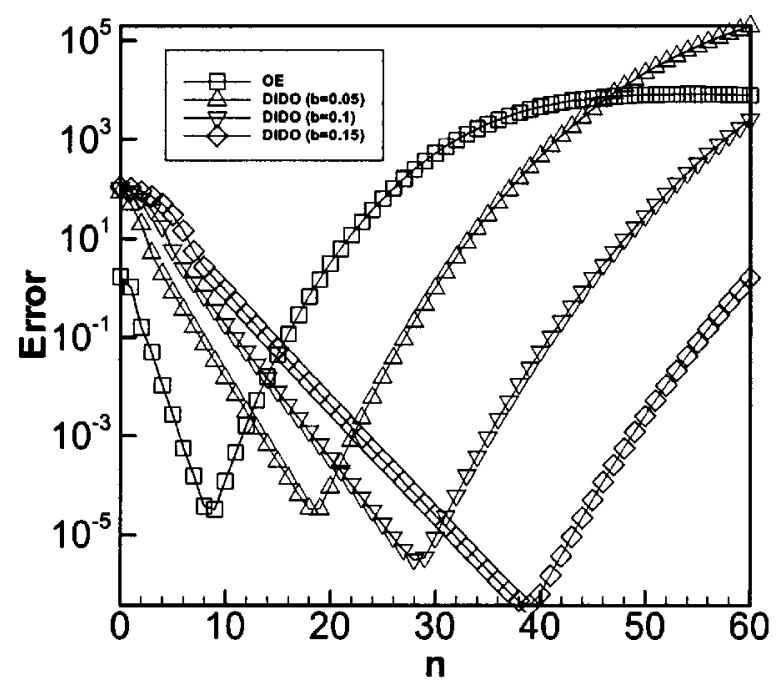

(a) Taylor

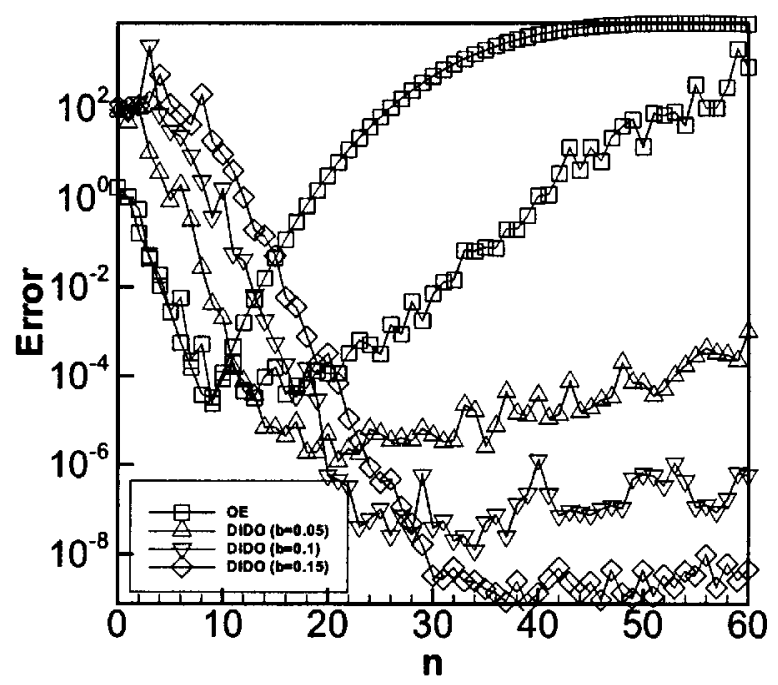

(b) Padé

Fig. 12. Error (3.16) in computation of the DNO by means of the OE and DIDO ( $b=0.05,0.1,0.15)$ algorithms for the sinusoidal profile (3.9) $\left(\lambda / d=0.4368, h / d=0.1, N_{x}=128, n=0, \ldots, 60\right)$. The results in (a) were obtained with Taylor summation, while those in (b) were obtained with Padé approximation.

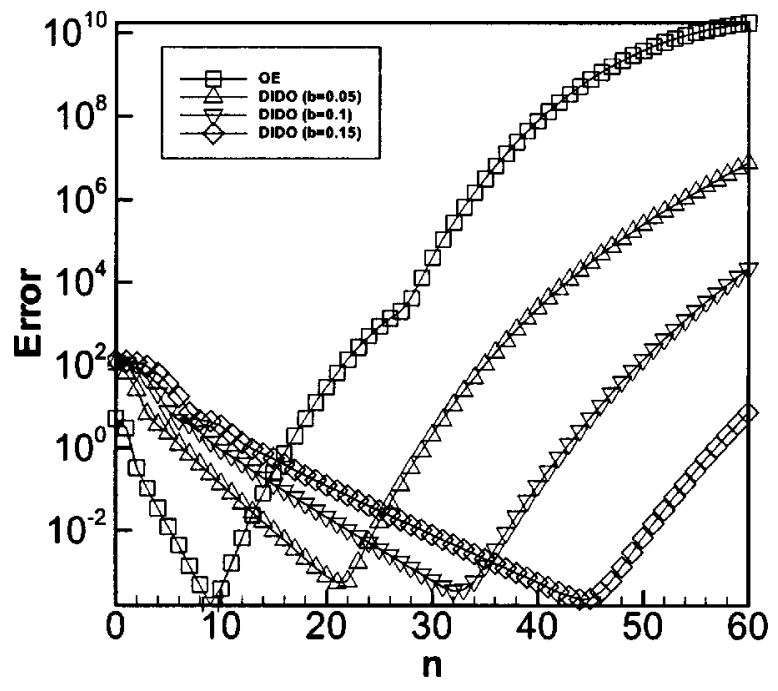

(a) Taylor

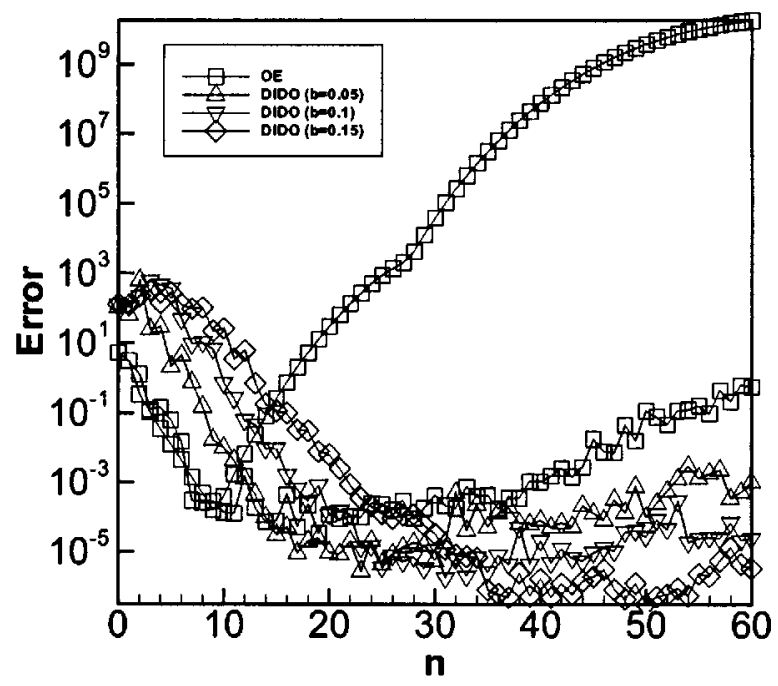

(b) Padé

Fig. 13. Error (3.16) in computation of the DNO by means of the OE and DIDO ( $b=0.05,0.1,0.15)$ algorithms for the rough profile (3.10) $\left(\lambda / d=0.4368, h / d=0.1, N_{x}=128, n=0, \ldots, 60\right)$. The results in (a) were obtained with Taylor summation, while those in (b) were obtained with Padé approximation.

the scattered fields to justify the use of the chain rule [e.g., in Eq. (2.3)]. In contrast, in the PTFE approach this justification is provided by the change of variables (3.1), which guarantees that the differentiations are always performed in the interior of the domain of definition of the fields [note that the argument of $v$ in Eq. (3.3) remains inside the domain $y>g(x)$ for all $y^{\prime}>0$ ]. This realization, in turn, suggests an alternative procedure that is based on mimicking this property (i.e., interior differentiations) while retaining the dimension-reducing benefits of the OE and PFE approaches.

To present these ideas, we begin by introducing a Dirichlet-to-interior-derivative operator (DIDO), which, much in the spirit of the DNO, maps Dirichlet data at the boundary $y=\delta g(x)$ to a "normal" derivative at a surface strictly inside the domain $y>\delta g(x)$ at

$$
y=b(1-\delta)+\delta g(x)=b-\delta[b-g(x)]
$$

where $b>0$ is to be chosen. More precisely, the DIDO $\widetilde{T}(\delta g)$ is defined as

$$
\begin{gathered}
\widetilde{T}(\delta g)[\xi](x)=\nabla v(x, b(1-\delta)+\delta g(x)) \\
\cdot\left(\delta \partial_{x} g(x),-1\right),
\end{gathered}
$$


where $v$ is the scattered outgoing (quasi-periodic) solution to the Helmholtz equation in $y>\delta g(x)$ with Dirichlet data $\xi(x)=v(x, \delta g(x))$.

When $\delta=1$, this DIDO coincides with the DNO, and thus it provides a way to perturbatively compute the latter while avoiding boundary differentiations:

$$
\begin{aligned}
T(g)[\xi] & =\left.\widetilde{T}(\delta g)[\xi]\right|_{\delta=1} \\
& =\left.\sum_{n=0}^{\infty}\left\{\widetilde{T}_{n}(g)[\xi]\right\} \delta^{n}\right|_{\delta=1} .
\end{aligned}
$$

Indeed, to derive a recurrence for the coefficients $\widetilde{T}_{n}(g)[\xi]$, we can proceed analogously to the derivation of Eq. (2.10). ${ }^{22}$ To this end, we begin by recalling that the functions in Eq. (3.14) provide exact solutions to the Helmholtz equation in $y>\delta g$, satisfy the Sommerfeld radiation condition, and are quasi-periodic, so that

$$
\begin{aligned}
\widetilde{T}(\delta g)\left[\operatorname { e x p } \left\{i \alpha_{p} x+\right.\right. & \left.\left.i \beta_{p} \delta g(x)\right\}\right] \\
= & \left\{\left[\delta \partial_{x} g(x)\right]\left(i \alpha_{p}\right)-i \beta_{p}\right\} \\
& \times \exp \left(i \alpha_{p} x+i \beta_{p}\{b-\delta[b-g(x)]\}\right) .
\end{aligned}
$$

Expanding in a series of powers of $\delta$ and equating like powers we obtain

$$
\begin{aligned}
& \widetilde{T}_{n}(g)\left[\exp \left(i \alpha_{p} x\right)\right] \\
& =\left[\partial_{x} g(x)\right]\left(i \alpha_{p}\right) \exp \left(i b \alpha_{p}\right)\left(i \beta_{p}\right)^{n-1} \frac{[b-g(x)]^{n-1}}{(n-1) !} \\
& \quad \times \exp \left(i \alpha_{p} x\right)-\exp \left(i b \alpha_{p}\right)\left(i \beta_{p}\right)^{n+1} \frac{[b-g(x)]^{n}}{n !} \\
& \quad \times \exp \left(i \alpha_{p} x\right)-\sum_{l=0}^{n-1} \widetilde{T}_{l}(g)\left[\left(i \beta_{p}\right)^{n-l}\right. \\
& \left.\quad \times \frac{\{g(x)\}^{n-l}}{(n-l) !} \exp \left(i \alpha_{p} x\right)\right] .
\end{aligned}
$$

Finally, defining $\beta_{D}$ as in Eq. (2.11) and

$$
\begin{aligned}
\exp \left(i b \alpha_{D}\right)\left[\sum_{p} \hat{\xi}_{p} \exp \left(i \alpha_{p} x\right)\right] & \\
& =\sum_{p} \exp \left(i b \alpha_{p}\right) \hat{\xi}_{p} \exp \left(i \alpha_{p} x\right),
\end{aligned}
$$

we arrive at

$$
\begin{aligned}
\widetilde{T}_{n}(g)[\xi]= & \partial_{x}\left\{\frac{[b-g(x)]^{n}}{n !} \partial_{x} \exp \left(i b \alpha_{D}\right)\left(i \beta_{D}\right)^{n-1} \xi\right\} \\
& +k^{2} \frac{[b-g(x)]^{n}}{n !} \exp \left(i b \alpha_{D}\right)\left(i \beta_{D}\right)^{n+1} \xi \\
& -\sum_{l=0}^{n-1} \widetilde{T}_{l}(g)\left[\frac{\{g(x)\}^{n-1}}{(n-l) !}\left(i \beta_{D}\right)^{n-l} \xi\right] .
\end{aligned}
$$

Our new scheme then will take advantage of the recurrence (3.18) to compute the DNO as in Eq. (3.17); from this, the scattered field can be computed as in the general OE framework (see Subsection 2.A).
We close this section with some numerical examples that illustrate the beneficial effects of the use of Eq. (3.18) in computing the DNO. As these examples demonstrate, the improvement of the DIDO over classical OE may be very significant, especially for a Fourier profile such as Eq. (3.9) or (3.10). And, although such impressive improvements may not be universally realized (especially for very rough surfaces), the potential accuracy gains come at no additional cost, which, we believe, should justify its use in rather general situations.

As we show below, and as is to be expected, the effectiveness of the DIDO scheme will generally depend upon the choice of the parameter $b$ (the case $b=0$ coincides, of course, with a standard OE algorithm). The optimal choice of this parameter is tied to the study of subtle issues of analytic continuation at $\delta=1$ (where the interior surface coincides with the boundary of the domain), which will be left for future work. For the present work, this optimization was performed numerically, as could be done in general. Indeed, a general strategy for the choice of this parameter could be based, for instance, on numerical minimization of the energy defect (3.12) or on observed convergence on a set of Rayleigh amplitudes, both of which, in fact, constitute common approaches in the absence of exact solutions.

Here, in Figs. 12 and 13, we display results corresponding to approximations of the currents (3.15) for $p=1$ with errors defined as in Eq. (3.16). The figures show this error as a function of $n$ for the profiles (3.9) and (3.10), respectively; in these experiments, $\lambda=0.4368, d$ $=1, \delta=0.05, N_{x}=128$, and $n$ varies from 0 to 60 .

Figure 12(a) illustrates the mechanism by which stability is enhanced if the parameter $b$ is chosen to be strictly positive: The interior differentiations provide a significant increase in the range of orders that can be accurately computed. Indeed, as $b$ increases from 0 , this effect is immediately apparent and it results in greatly improved values. As $b$ is further increased, however, the results begin to deteriorate again as a consequence of the fact that the boundary $y=b$ at $\delta=0$ is increasingly far from the target boundary $y=g(x)$ at $\delta=1$. Indeed, in this case, the perturbation series begins to converge too slowly to benefit from the increase in the range of stable orders $n$. In any event, for suitable values of $b$, this increase has a rather dramatic effect that is, in fact, further magnified if Padé approximants are used to evaluate the series; see Fig. 12(b). In fact, as we show in Figs. 13(a) and 13(b), Padé approximation will typically reveal this enhanced stability even if it is not apparent in a comparison of Taylor sums. Again here, the reason lies in the increased range of orders that can be stably evaluated when $b>0$ [see Fig. 13(a)], which can be exploited by the Padé procedure independently of the radius of convergence of the Taylor series.

\section{CONCLUDING REMARKS}

The discussion and the numerical examples presented here clearly demonstrate that the principles derived from the theories in the first part of this work ${ }^{22}$ can be realized in practice to derive shape-deformation algorithms with improved conditioning properties. Indeed, our new re- 
sults provide further evidence that the stability properties of these methods are governed by cancellations in the underlying recursive relations for the functional coefficients of the perturbation expansion. And they confirm the contention in Ref. 22 that stabilized algorithms should result from appropriate management of such cancellations. As suggested in Ref. 22, this management can be explicitly attained in low-order implementations, where cancellations can be mitigated through a careful but explicit recasting of the formulas. For high-order expansions, on the other hand, the increasing complexity of the expressions conspires against an explicit accounting. As we have shown, however, the insight gained in Ref. 22 can be used to design a number of strategies to implicitly mollify cancellations, resulting in numerical algorithms with greatly enhanced stability properties. In particular, we have introduced two distinct approaches to improve upon the classical field expansion (FE) and operator expansion $(\mathrm{OE})$ formalisms, respectively. In relation to the FE scheme, our improved procedure of partial transformed field expansion (PTFE) relies on the observation ${ }^{22}$ that cancellations can be avoided if a change of independent variables is effected in advance of the derivation of the corresponding recurrence. As we observed, a key feature of this change of variables is that it avoids differentiation across the boundary of the domain of definition of the fields when the recursion is being derived. This, in turn, motivated our improvement of the OE method wherein the avoidance of such boundary differentiations is attained by computing the Dirichlet-to-Neumann operator (DNO) by analytic continuation on a family of Dirichlet-to-interior-derivative operators (DIDOs). In connection with these new high-order implementations, interesting questions remain, including the search for better adapted bases for PTFE and for a theoretically optimal value of the interior parameter [b; cf. Eq. (3.18)] in the DIDO. In any case, the results presented here clearly place the new algorithms (both low- and highorder versions) on a competitive ground with classical schemes, and, in fact, they suggest that they may be, in many instances, preferable over standard implementations.

\section{APPENDIX A: CANCELLATIONS IN LOW- ORDER OPERATOR EXPANSION AND PARTIAL FIELD EXPANSION FORMULAS}

For the sake of completeness, in this appendix we present the derivation of the basic results that allow us to identify cancellations in low-order formulas. At first order, we recall Eq. (2.13a) and establish the following:

Theorem A.1. Let $f(x)$ be a $d$-periodic and Lipschitzcontinuous function, let $\eta(x)$ be quasi-periodic and square-integrable on $[0, d]$, and define $R_{f}[\eta]$ as in Eq. (2.15). Then, $R_{f}[\eta]$ is square-integrable on $[0, d]$, and, if

$$
R_{f}[\eta](x)=\sum_{p} \hat{R}_{p} \exp \left(i \alpha_{p} x\right)
$$

is the Fourier expansion of $R_{f}[\eta]$, we have

$$
\hat{R}_{p}=\sum_{q} \frac{[-(2 \pi / d) q]\left(\alpha_{p}+\alpha_{p-q}\right)}{\beta_{p}+\beta_{p-q}} \hat{f}_{q} \hat{\eta}_{p-q} .
$$

Proof. The proof of the square-integrability of $R_{f}[\eta]$ is presented in Appendix A of Ref. 22 and will be omitted here. Formula (A1) is established explicitly by using the Fourier representation of $f$ and $\eta$, and Eq. (2.17). To this end, first note that

$$
\begin{aligned}
\alpha_{p}^{2}+\beta_{p}^{2} & =\alpha_{p-q}^{2}+\beta_{p-q}^{2}=k^{2}, \\
\alpha_{p}-\alpha_{p-q} & =(2 \pi / d) q,
\end{aligned}
$$

so that

$$
\beta_{p}-\beta_{p-q}=\frac{\left(\alpha_{p}+\alpha_{p-q}\right)[-(2 \pi / d) q]}{\beta_{p}+\beta_{p-q}} .
$$

On the other hand, from Eq. (2.17), we have

$$
\hat{R}_{p}=\sum_{q}\left(\beta_{p}-\beta_{p-q}\right) \hat{f}_{q} \hat{\eta}_{p-q}
$$

and the representation (A1) by simply substituting Eq. (A2) into this latter relation.

The second-order result concerns cancellations that arise in the operator $T_{2}$ as well as in $v_{2}$ and $v_{3}$; see Eqs. $(2.13 \mathrm{~b})$ and (2.14).

Theorem A.2. Let $f(x)$ and $\eta$ be as in Theorem A.1 and define $S_{f}[\eta]$ as in Eq. (2.16). Then the function $\beta_{D} S_{f}[\eta]$ is square-integrable on $[0, d]$ and, if

$$
S_{f}[\eta](x)=\sum_{p} \hat{S}_{p} \exp \left(i \alpha_{p} x\right)
$$

is the Fourier expansion of $S_{f}[\eta]$, we have

$$
\begin{aligned}
\hat{S}_{p}= & \sum_{r} \sum_{q}\left[\frac{(2 \pi / d)^{2} q r}{\beta_{p}+\beta_{p-r-q}}\right] \\
& \times\left[1+\frac{\left(\alpha_{p}+\alpha_{p-q}\right)\left(\alpha_{p-q}+\alpha_{p-r-q}\right)}{\left(\beta_{p}+\beta_{p-q}\right)\left(\beta_{p-q}+\beta_{p-r-q}\right)}\right] \hat{\eta}_{p-r-q} \hat{f}_{r} \hat{f}_{q} .
\end{aligned}
$$

Proof. The proof of the square-integrability of $\beta_{D} S_{f}[\eta]$ is again contained in Appendix A of Ref. 22 and will be omitted. To establish Eq. (A3), we first note that from Eq. (2.18) we have

$$
\begin{aligned}
\hat{S}_{p}= & \frac{1}{2} \sum_{r} \sum_{q} \hat{f}_{r} \hat{f}_{q} \hat{\eta}_{p-r-q}\left[\left(\beta_{p-q}+\beta_{p}\right)\right. \\
& \left.+\left(\beta_{p-q}-\beta_{p-r-q}\right)\right]
\end{aligned}
$$

which, with the use of Eq. (A2), can be written as

$$
\begin{aligned}
\hat{S}_{p}= & \frac{1}{2} \sum_{r} \sum_{q} \hat{f}_{r} \hat{f}_{q} \hat{\eta}_{p-r-q}\left\{\frac{[(2 \pi / d) q]\left(\alpha_{p}+\alpha_{p-q}\right)}{\beta_{p}+\beta_{p-q}}\right. \\
& \left.+\frac{[-(2 \pi / d) r]\left(\alpha_{p-q}+\alpha_{p-r-q}\right)}{\beta_{p-q}+\beta_{p-r-q}}\right\} .
\end{aligned}
$$


Note that the last expression still entails significant cancellations, as follows from its symmetries: For instance, for large values of $p$ we have that the sum behaves according to

$$
\approx \frac{1}{2} \sum_{r} \sum_{q} \hat{f}_{r} \hat{f}_{q} \hat{\eta}_{p-r-q}(2 \pi / d)^{2}\left(\frac{2 q \alpha_{p}}{2 \beta_{p}}+\frac{-2 r \alpha_{p}}{2 \beta_{p}}\right)=0 .
$$

To derive the stable, cancellation-free formula (A3) from Eq. (A4), it will be convenient to introduce the quantities

$$
\begin{aligned}
A & =\beta_{p}+\beta_{p-q}, \quad B=\beta_{p-q}+\beta_{p-r-q}, \\
C & =\beta_{p}+\beta_{p-r-q}, \\
a & =[(2 \pi / d) q]\left(\alpha_{p}+\alpha_{p-q}\right), \\
b & =[-(2 \pi / d) r]\left(\alpha_{p-q}+\alpha_{p-r-q}\right),
\end{aligned}
$$

in terms of which Eq. (A4) takes on the form

$$
\hat{S}_{p}=\frac{1}{2} \sum_{r} \sum_{q} \hat{f}_{r} \hat{f}_{q} \hat{\eta}_{p-r-q}\left(\frac{a}{A}+\frac{b}{B}\right) .
$$

Then, using

$$
\frac{1}{A}-\frac{1}{C}=\frac{-b}{A B C}, \quad \frac{1}{B}-\frac{1}{C}=\frac{-a}{A B C},
$$

we can write

$$
\begin{aligned}
\hat{S}_{p} & =\frac{1}{2} \sum_{r} \sum_{q} \hat{f}_{r} \hat{f}_{q} \hat{\eta}_{p-r-q}\left(\frac{a}{C}-\frac{a b}{A B C}+\frac{b}{C}-\frac{a b}{A B C}\right) \\
& =\frac{1}{2} \sum_{r} \sum_{q} \hat{f}_{r} \hat{f}_{q} \hat{\eta}_{p-r-q}\left(\frac{a+b}{C}-\frac{2 a b}{A B C}\right) \\
& =\frac{1}{2} \sum_{r} \sum_{q} \hat{f}_{r} \hat{f}_{q} \hat{\eta}_{p-r-q}\left[\frac{(2 \pi / d)^{2} 2 q r}{C}-\frac{2 a b}{A B C}\right],
\end{aligned}
$$

where we have used the symmetries in $r$ and $q$ to replace

$$
\begin{aligned}
a+b= & (2 \pi / d) 2 \alpha(q-r)+(2 \pi / d)^{2}\{q(2 p-q) \\
& -r[2(p-q)-r]\} \text { by }(2 \pi / d)^{2} 2 q r .
\end{aligned}
$$

Finally, since

$$
2 a b=-2(2 \pi / d)^{2} q r\left(\alpha_{p}+\alpha_{p-q}\right)\left(\alpha_{p-q}+\alpha_{p-r-q}\right),
$$

it follows that

$$
\begin{aligned}
\hat{S}_{p}= & \sum_{r} \sum_{q} \hat{f}_{r} \hat{f}_{q} \hat{\eta}_{p-r-q}\left[\frac{(2 \pi / d)^{2} q r}{C}\right] \\
& \times\left[1+\frac{\left(\alpha_{p}+\alpha_{p-q}\right)\left(\alpha_{p-q}+\alpha_{p-r-q}\right)}{\left(\beta_{p}+\beta_{p-q}\right)\left(\beta_{p-q}+\beta_{p-r-q}\right)}\right],
\end{aligned}
$$

which immediately delivers Eq. (A3).

\section{ACKNOWLEDGMENTS}

David P. Nicholls gratefully acknowledges support from the National Science Foundation (NSF) through grants DMS-0196452 and DMS-0139822. Fernando Reitich gratefully acknowledges support from the NSF through grants DMS-9971379 and DMS-0311763, from the U.S. Air Force Office of Scientific Research through contract
F49620-02-1-0052, and from the U.S. Army High Performance Computing Research Center (AHPCRC) under Army Research Laboratory cooperative agreement DAAD19-01-2-0014.

Disclaimer. Effort sponsored by the Air Force Office of Scientific Research, Air Force Materials Command, USAF, under grant F49620-02-1-0052 and by the AHPCRC under the auspices of the Department of the Army, Army Research Laboratory cooperative agreement DAAD19-01-2-0014. The U.S. Government is authorized to reproduce and distribute reprints for governmental purposes notwithstanding any copyright notation thereon. The views and the conclusions contained herein are those of the author and should not be interpreted as necessarily representing the official policies or endorsements, either expressed or implied, of the Air Force Office of Scientific Research, the Army Research Laboratory, or the U.S. Government.

The authors' e-mail addresses are Nicholls.2@nd.edu and reitich@math.umn.edu.

\section{REFERENCES}

1. Lord Rayleigh, "On the dynamical theory of gratings," Proc. R. Soc. London Ser. A 79, 399-416 (1907).

2. S. O. Rice, "Reflection of electromagnetic waves from slightly rough surfaces," Commun. Pure Appl. Math. 4, 351-378 (1951).

3. G. V. Anand and M. K. George, "Normal mode sound propagation in an ocean with sinusoidal surface waves," J. Acoust. Soc. Am. 80, 238-243 (1986).

4. G. V. Anand and M. K. George, "Normal mode sound propagation in an ocean with random narrow-band surface waves," J. Acoust. Soc. Am. 94, 279-292 (1993).

5. E. Y. Harper and F. M. Labianca, "Perturbation theory for scattering of sound from a point source by a moving rough surface in the presence of refraction," J. Acoust. Soc. Am. 57, 1044-1051 (1975).

6. E. Y. Harper and F. M. Labianca, "Scattering of sound from a point source by a rough surface progressing over an isovelocity ocean," J. Acoust. Soc. Am. 58, 349-364 (1975).

7. W. A. Kuperman and F. F. Ingenito, "Attenuation of the coherent component of sound propagating in shallow water with rough boundaries," J. Acoust. Soc. Am. 61, 1178-1187 (1977).

8. A. H. Nayfeh and O. R. Asfar, "Parallel-plate waveguide with sinusoidally perturbed boundaries," J. Appl. Phys. 45, 4797-4800 (1974).

9. J. R. Wait, "Perturbation analysis for reflection from twodimensional periodic sea waves," Radio Sci. 6, 387-391 (1971).

10. J. M. Chesneaux and A. A. Wirgin, "Response to comments on 'Reflection from a corrugated surface revisited," J. Acoust. Soc. Am. 98, 1815-1816 (1995).

11. J. J. Greffet, "Scattering of electromagnetic waves by rough dielectric surfaces," Phys. Rev. B 37, 6436-6441 (1988).

12. J. J. Greffet, C. Baylard, and P. Versaevel, "Diffraction of electromagnetic waves by crossed gratings: a series solution," Opt. Lett. 17, 1740-1742 (1992).

13. J. J. Greffet and Z. Maassarani, "Scattering of electromagnetic waves by a grating: a numerical evaluation of the iterative-series solution,” J. Opt. Soc. Am. A 7, 1483-1493 (1990).

14. D. R. Jackson, D. P. Winebrenner, and A. Ishimaru, "Comparison of perturbation theories for rough-surface scattering," J. Acoust. Soc. Am. 83, 961-969 (1988).

15. L. Kazandjian, "Comparison of the Rayleigh-Fourier and extinction theorem methods applied to scattering and 
'transmission at a rough solid-solid interface," J. Acoust. Soc. Am. 92, 1679-1691 (1992).

16. C. Lopez, F. J. Yndurain, and N. Garcia, "Iterative series for calculating the scattering of waves from hard corrugated surfaces," Phys. Rev. B 18, 970-972 (1978).

17. A. A. Maradudin, "Iterative solutions for electromagnetic scattering by gratings," J. Opt. Soc. Am. 73, 759-764 (1983).

18. J. Roginsky, "Derivation of closed-form expressions for the $T$ matrices of Rayleigh-Rice and extinction-theorem perturbation theories," J. Acoust. Soc. Am. 90, 1130-1137 (1991).

19. V. I. Tatarskii, "Relation between the Rayleigh equation in diffraction theory and the equation based on Green's formula," J. Opt. Soc. Am. A 12, 1254-1260 (1995).

20. A. Wirgin, "Scattering from hard and soft corrugated surfaces: iterative corrections to the Kirchhoff approximation through the extinction theorem," J. Acoust. Soc. Am. 85, 670-679 (1989).

21. O. P. Bruno and F. Reitich, "Solution of a boundary value problem for the Helmholtz equation via variation of the boundary into the complex domain," Proc. R. Soc. Edinburgh, Sect. A 122, 317-340 (1992).

22. D. P. Nicholls and F. Reitich, "Shape deformations in rough surface scattering: Cancellations, conditioning, and convergence," J. Opt. Soc. Am. A 21, 590-605 (2004).

23. D. M. Milder, "The effects of truncation on surface-wave Hamiltonians," J. Fluid Mech. 217, 249-262 (1990).

24. D. M. Milder, "An improved formalism for rough-surface scattering of acoustic and electromagnetic waves," in Wave Propagation and Scattering in Varied Media II, V. Varadan, ed., Proc. SPIE 1558, 213-221 (1991).

25. D. M. Milder, "An improved formalism for wave scattering from rough surfaces," J. Acoust. Soc. Am. 89, 529-541 (1991).

26. D. M. Milder and H. T. Sharp, "Efficient computation of rough surface scattering," in Mathematical and Numerical Aspects of Wave Propagation Phenomena (Society for Industrial and Applied Mathematics, Philadelphia, Pa., 1991), pp. 314-322.
27. D. M. Milder and H. T. Sharp, "An improved formalism for rough surface scattering. II: Numerical trials in three dimensions," J. Acoust. Soc. Am. 91, 2620-2626 (1992).

28. D. M. Milder, "Role of the admittance operator in roughsurface scattering," J. Acoust. Soc. Am. 100, 759-768 (1996).

29. D. M. Milder, "An improved formalism for electromagnetic scattering from a perfectly conducting rough surface," Radio Sci. 31, 1369-1376 (1996).

30. A. P. Calderón, "Cauchy integrals on Lipschitz curves and related operators," Proc. Natl. Acad. Sci. USA 75, 13241327 (1977).

31. R. Coifman and Y. Meyer, "Nonlinear harmonic analysis and analytic dependence," in Pseudodifferential Operators and Applications (American Mathematical Society, Providence, R.I., 1985), pp. 71-78.

32. C. Canuto, M. Y. Hussaini, A. Quarteroni, and T. A. Zang, Spectral Methods in Fluid Dynamics (Springer-Verlag, New York, 1988).

33. D. Gottlieb and S. A. Orszag, Numerical Analysis of Spectral Methods: Theory and Applications (Society for Industrial and Applied Mathematics, Philadelphia, Pa., 1977).

34. R. Petit, ed., Electromagnetic Theory of Gratings (SpringerVerlag, Berlin, 1980).

35. O. P. Bruno and F. Reitich, "Numerical solution of diffraction problems: a method of variation of boundaries," J. Opt. Soc. Am. A 10, 1168-1175 (1993).

36. O. P. Bruno and F. Reitich, "Numerical solution of diffraction problems: a method of variation of boundaries. II. Finitely conducting gratings, Padé approximants, and singularities,” J. Opt. Soc. Am. A 10, 2307-2316 (1993).

37. O. P. Bruno and F. Reitich, "Numerical solution of diffraction problems: a method of variation of boundaries: III. Doubly periodic gratings,” J. Opt. Soc. Am. A 10, 2551-2562 (1993).

38. D. P. Nicholls and F. Reitich, "Stability of high-order perturbative methods for the computation of Dirichlet-Neumann operators," J. Comput. Phys. 170, 276-298 (2001).

39. D. P. Nicholls and F. Reitich, "Analytic continuation of Dirichlet-Neumann operators," Numer. Math. 94, 107-146 (2003). 